\title{
Form und Funktion von Kasus bei Agrammatismus*
}

\author{
Josef Bayer, Nijmegen, Ria De Bleser und Claudia Dronsek, Aachen
}

„Beim Fressen beim Fernsehen fält der Vater dem Kartoffel aus dem Maul“ (Titel eines Romans von Eckard Henscheid)

\section{$1 \quad$ Einleitung}

Ziel der vorliegenden Untersuchungen zum deutschen Kasussystem und dessen Beeinträchtigung bei Aphasie ist, gewisse aphasische Störungen linguistisch genauer zu charakterisieren. Wie wir zeigen werden, haben solche Charakterisierungen weitreichende Konsequenzen, u.a. für das Verständnis eines normalen, d.h. nicht gestörten, Sprachverarbeitungssystems. Andererseits versuchen wir zu zeigen, welche Konsequenzen sich aus unserer Interpretation aphasischer Störungen für das Verhältnis von Syntax und Lexikon ergeben.

Der Anstoßzu den Untersuchungen wurde bereits vor Jahren gegeben durch Heeschens Behauptung, daß Agrammatiker diejenigen Äußerungen, die sie hervorbringen, in der Regel wohl strukturieren. Nach Heeschens Ansicht erscheint zwar die agrammatische Sprache gestört, aber die Phrasen, die auftreten, sind in sich wohl geformt. Die Beispiele, auf die sich diese Behauptung bezog, waren von der folgenden Art: Wenn ein Agrammatiker die Proposition ,der dicke Mann trinkt Kaffee" äußern will, so ergeben sich - je nach Schweregrad der Aphasie - verschiedene Möglichkeiten der Realisation, während andere logisch denkbare Möglichkeiten ausscheiden. Der Asterisk steht im folgenden für ,Unagrammatikalität“":

$$
\text { Mann ... dick ... Mann ... Kaffee }
$$

(schwere Störung)

Dicker Mann ... Kaffee trinken

(mittlere Störung)

(4)

Der dicke Mann trinkt Kaffee

(leichte Störung)

(5)

$$
\text { *Dickes Mann Kaffee trinkt }
$$

*Dick Mann trinken Kaffee

* Die Forschungen, die zu diesem Aufsatz geführt haben, wurden teilweise durch die Deutsche Forschungsgemeinschaft unterstützt (Projekt Po 41/16-1). Ein Teil der Ergebnisse wurde auf Vorträgen in Wien, Aachen und Düsseldorf, sowie auf der Academy of Aphasia in Nashville, Tennessee (Oktober 1986) präsentiert. Wir danken den Zuhörern, die uns durch ihre Anregungen unterstützt haben, sowie Angela Friederici für ihre Anmerkungen zum Text. 
Die Heeschen'schen Voraussagen für die agrammatische Sprachstörung stellen s:?. siarke und deshalb empirisch interessante Beschränkung des Defizits zur Dehatte. die nach einer Erklärung verlangt. Sollte es in der Tat so sein, daß Agrammatiker Außerungen des Typs (1)-(3), nicht aber des Typs (4), (5) machen -- was wir nicht systematisch untersucht haben -, welche Schlüsse sollte man daraus für die Vatur der Störung ziehen? Wir sind der Ansicht, daß solche Daten allein überhaupt nichts sagen, d.h. daß sie nur auf dem Hintergrund einer Theorie beginnen, ,.Mitteilungen “ zu machen. Die Theorie, die wir zugrundelegen, ist im wesentlichen eine repräsentationale. Da wir nicht genau wissen, wie Sprache produziert wird und wie weit die Sprachproduktion von grammatischer Komplexität beeinflußt wird, nehmen wir zwei Einschränkungen vor:

(a) Wir benutzen spontan hervorgebachte Sprache nur als Heuristik zur Auswahl von Versuchspersonen, halten uns aber ansonsten an experimentell elizitierte Daten:

(b) wir interpretieren die Daten auf dem Hintergrund einer repräsentationalen linguistischen Theorie, die uns geeignet und in sich entwickelt genug erscheint, um einen Teil des sprachlichen Kenntnissystems auf interessante Weise zu charakterisieren.

Beide Einschränkungen sollen uns nicht hindern, Spekulationen über die Produktion von Spontansprache und über Sprachverarbeitung allgemein anzubringen, wenn es erforderlich ist.

Der Aufsatz ist wie folgt aufgebaut: In Paragraph 2 werden einige Ansätze zur Charakterisierung der agrammatischen Sprachstörung diskutiert. In 3 führen wir die linguistischen Begriffe ein, die uns für die Besprechung des Kasussystems relevant erscheinen. Die Patienten, die an den Untersuchungen beteiligt waren, werden in 4 im einzelnen vorgestellt. Sektion 5 berichtet die Experimente und die Ergebnisse. In der Schlußdiskussion (Sektion 6) fassen wir diese Ergebnisse zusammen und stellen dar, welche Schlüsse daraus sowohl für die linguistische Theorie als auch für die neuropsychologische Charakterisierung gewisser Aphasien gezogen werden können.

\section{Ansätze zur Charakterisierung der agrammatischen Sprach- störung(en)}

Rein observationell ist die agrammatische Sprachstörung dadurch charakterisiert, daß Sprache mühevoll und nur in kleineren Fragmenten hervorgebracht wird. Statt flektierter Wortformen werden oft unmarkierte Formen benutzt, z. B. der Nominativ, der Infinitiv, die unflektierte Form des Adjektivs etc.; Elemente der ,Funktionswort"-Klasse fallen der Tendenz nach wesentlich häufiger aus als Elemente der ,.Inhaltswort"“Klasse. Es scheint. als ob ein Ökonomieprinzip herrsche, das dem Patienten diktiert, nur die allernötigsten Bausteine der Rede zu äußern, die eine semantische Interpretation oder ein Erschließen der Sprecherabsicht erlaubt. Der klinische Eindruck ist gewöhnlich der, daß Agrannma- 
tiker unvergleichlich besser verstehen als produzieren. Manchmal wird sogar berichtet, daß einige solche Patienten perfekte Versteher sind ${ }^{1}$. Meistens zeigen sich allerdings gewisse Sprachverständnisstörungen, besonders dann, wenn die Information nicht durch situative/kontextuelle Faktoren redundant angeboten wird, bzw. wenn die Informationen sprachlich ,komplex“ enkodiert ist. So können beispielsweise Agrammatiker in der Regel Anweisungen wie diese aus dem fünften Teil des Token Tests nicht korrekt interpretieren: „Bevor Sie den grünen Kreis berühren, nehmen Sie das weiße Viereck“, während sie keine Probleme haben mit Anweisungen wie ,öffnen Sie die Tür und das Fenster" oder bei Mehrfachwahlaufgaben, bei denen zu einem Satz das passende aus vier Bildern auszuwählen ist.

Agramınatiker haben häufig, wenn sie eine Läsion im prä-rolandischen Operculum haben, eine bracchiofaziale Lähmung und eine Dysarthrie. ${ }^{2}$ Bei solchen Patienten ist es manchmal schwierig, differential-diagnostisch festzustellen, wieviel Anteil an der Störung auf periphere Prozesse entfällt und, wenn überhaupt, wieviel auf das zentrale Sprachverarbeitungssystem entfällt. In der Regel gibt aber die korrekte Durchführung eines standardisierten Tests wie des Aachener Aphasie Tests ${ }^{3}$ darüber Auskunft, ob eine Aphasie vorliegt und von welchem Schweregrad sie ist. Wir wollen hier nicht weiter auf die verschiedenen Modalitäten (z. B. Lesen, Schreiben, Benennen etc.) eingehen, die beim Vorliegen einer agrammatischen Störung beeinträchtigt sein können ${ }^{4}$, sondern sofort dazu übergehen, einige prominente Erklärungsansätze vorzustellen.

\subsection{Agrammatismus als Syntaxstörung}

Die vorherrschende Meinung in der amerikanischen Aphasiologie der 70er Jahre war, dak dem Agrammatismus eine zentrale Syntaxstörung zugrundeliegt, die durch die Läsion einer corticalen Region hervorgerufen ist, in der beim Menschen ein syntaktischer Parser lokalisiert ist. Bei einer entsprechenden Schädigung wäre keinerlei syntaktisches Verarbeiten mehr möglich. Camarazza und Zurif versuchten das durch Experimente zu erhärten, indem sie u.a. Agrammatikern ,Satzlegeaufgaben" stellten, bei denen die Patienten nach einer von Levelt enwickelten Methode Satzkonstituenten nach ihrer "Zusammengehörigkeit" evaluieren müssen ${ }^{5}$. Die Ergebnisse zeigten, daß die Patienten im wesentlichen nur die "Inhaltswörter" also volle Nomina, Verben außer Hilfsverben und Adjektive, beachten und die Elemente, die die syntaktische Verklammerung leisten, also Artikel, Präpositionen usw., nicht adäquat an die syntaktischen Hauptkategorien binden. Vergleichbare Resultate erbrachten Verständnistests, bei denen die Patienten zu einem vorgegebenen Satz aus einer Menge von damit semantisch relatierten Bildern das (syntaktisch) passende auszuwählen hatten. Agrammatiker fielen dabei genau dann herein, wenn die Erschließung der Bedeutung nicht durch sog. ,,semantische Heuristiken“ möglich war, sondern syntaktisches Verarbeiten essentiell voraussetzte. Weitere Experimente zeigten, daß3 Agrammatiker Prinzipien der Wortfolge nicht oder nur unzureichend zur Verfügung haben und sich bereits bei der Interpretation einfacher englischer SVO-Sätze zu Strategien flüchten, wie etwa die erste NP als Agens zu deuten, was 
dann bei der Bearbeitung von Passivsätzen oder von semantisch reversiblen Sätzen (z.B. "The triangle shoots the square") zu Fehlern führt ${ }^{6}$. Solche und weitere Ergebnisse dieser Art verfestigten die Meinung, daß die agrammatische Sprachstörung durch einen weitgehenden, wenn nicht vollständigen Verlust der Fähigkeit zu charakterisieren ist, Äußerungen eine syntaktische Analyse zu geben.

\subsection{Der gestörte Zugriff zu Elementen der „geschlossenen Klasse“}

Eng damit verbunden sind Forschungsrichtungen, die Evidenz dafür gebracht haben, daß Agrammatiker die Elemente der sog. "closed class" nicht wie Normalpersonen verarbeiten. So hat Bradley (1978) in Experimenten gezeigt, daß lexikalische Entscheidungszeiten für Wörter der offenen Klasse eine Funktion der Wortfrequenz sind, daß hingegen ein Frequenzeffekt für Wörter der geschlossenen Klasse nicht existiert ${ }^{7}$. Bradley, Garrett \& Zurif (1980) haben gezeigt, daß anders als Normale - Agrammatiker für beide Vokabular-Typen gleiche Verarbeitungsmechanismen einzusetzen scheinen. Unabhängig davon existieren Daten aus der Fehlerlinguistik, die nahelegen, daß das Funktionswortvokabular bei der Sprachproduktion zu einem früheren Zeitpunkt als eine Art Satzrahmen vorhanden ist als das Inhaltswortvokabular, das in diese Rahmen eingesetzt wird ${ }^{8}$. Man kann die Verarbeitungsresultate für Agrammatiker so deuten, daß das für die Erstellung von syntaktischen Strukturen nötige Vokabular nicht in Realzeit produziert werden kann ${ }^{9}$.

Die Idee des gestörten Zugriffs zu einem bestimmten ausgewählten Vokabular setzte sich in der anglo-amerikanischen Aphasiologie breit durch. Es sei angemerkt. daß die empirische Grundlage dazu fast ausschließlich Experimente mit englischsprachigen Patienten bildeten. d.h. daß ein sprachspezifischer Effekt dabei nicht von vorneherein auszuschließen ist. Kean (1977) hat schließlich versucht, dars postulierte Defizit linguistisch konsistent als phonologische Störung zu beschreiben. Kean kam es im wesentlichen darauf an, mit Hilfe der phonologischen Theorie von Chomsky \& Halle (1968) ein Kriterium anzugeben, nach dem bestimnte Morphemgruppen unterschieden werden können. Das zentrale Kriterium für die linguistische Beschreibung des Agrammatismus ist nach Keans (damaliger) Argumentation der Status des "phonologischen Worts". Ein phonologisches Wort ist eine Kette von Segmenten (Phonemen), die durch Grenzsymbole flankiert ist. welche in der Akzentzuweisung eine Rolle spielen. Sogenannte "word boundary". Morpherne affizieren das Akzentmuster eines Wortes nicht; es gibt aber andere Morpheme, die das wohl tun, vgl. im Englischen définite/définitness versus définite definitive. Im allgemeinen beeinflußen Flexionsaffixe das Akzentmuster des Wortes oder Stammes nicht, an den sie treten, z. B. gárden/gárden-s, regrét/regrét-s usw Kean hat nun vorgeschlagen, daß Agrammatiker (bei ihr: Broca-Aphasiker) dazı tendieren, Sätze auf die minimale Kette von Wörtern zu reduzieren, die als phonologische Wörter in der jeweiligen Sprache konstruierbar sind. Während Wortbildungsaffixe wie -ive oder per- durch schwache Grenzsymbole $(+)$ vom Stammorphe?.. getrennt sind, sind die lexikalischen Hauptkategorien, die als freie Morpheme auf- 
treten können, durch starke Grenzen (\#) flankiert, also z. B. [A \# definit + ive \#], $[\mathrm{v} \#$ per + mit $\#]$ versus $[\mathrm{N} \#[\mathrm{~N} \#$ boy $\#] \mathrm{s} \#]$. Betrachten wir nun die Voraussagen für eindeutige Flexionssuffixe im Deutschen, so müßte ein Satz wie [DET der] $\left[\mathrm{A}_{\mathrm{A}} \#\left[\mathrm{~A}_{\mathrm{A}} \# \operatorname{dick} \#\right] \mathrm{e} \#\right][\mathrm{N} \# \operatorname{mann} \#][\mathrm{V} \#[\mathrm{~V} \# \operatorname{trink} \#] \mathrm{t} \#][\mathrm{DET} \operatorname{den}]\left[_{\mathrm{A}} \#\left[\mathrm{a}_{\mathrm{A}} \#\right.\right.$ [A $\#$ heiss \#] e\#] $\mathrm{n} \#$ ] [N $\#$ kaffee \#] reduziert werden zu dick Mann trink heiß Kaffee. Dies entspricht nun mit ziemlicher Sicherheit keiner agrammatischen Äußerung, wie selbst Untersucher mit nur geringer klinischer Erfahrung wissen ${ }^{10}$.

Wir halten dies für ein Indiz, daß zumindest eine monokausale Erklärung des Agrammatismus als Störung des Zugriffs zu Elementen der ,geschlossenen Klasse“ bzw. als phonologische Störung im Sinne von Kean nicht richtig sein kann.

\subsection{Agrammatismus als reine Performanstörung}

Der Gedanke, Agrammatismus als Performanzstörung zu beurteilen, ist etwa so alt wie die Chomsky'sche Theorie von Kompetenz und Performanz. Bereits Lenneberg (1972) streicht den Zeitfaktor als die ,,bedeutsamste Dimension in der Sprachphysiologie" heraus ${ }^{11}$. Weigl \& Bierwisch (1970) gehen explizit davon aus, daß Aphasien - mit Ausnahme der globalen Aphasie - Performanzstörungen sind und die Sprachkompetenz unberührt lassen. Die Evidenz für diese Ansicht ist natürlich davon abhängig, inwieweit die Unterscheidung von Kompetenz und Performanz in der Neuropsychologie fruchtbar, d.h. präzise, gemacht werden kann. Lassen wir dieses weitschweifige Thema beiseite und wenden wir uns einem neueren Beispiel zu, das zum Zweck der Einführung andere mitrepräsentieren könnte. Linebarger, Schwartz \& Saffran (1983) behaupten, daß sogenannte „Agrammatiker“ überhaupt keine gestörte Syntax haben und daß sie nur deshalb agrammatisch sprechen und verstehen, weil sie syntaktische Repräsentationen nicht für die semantische Interpretation ausnüzen können oder aber weil sie syntaktisches Verarbeiten nur auf Kosten des semantischen Verarbeitens leisten können und umgekehrt. Diese Erklärung des Agrammatismus lastet die Störung ausschließlich den Sprachverarbeitungsmechanismen auf und nimmt zugleich ein vollständiges oder weitgehend erhaltenes System der syntaktischen wie semantischen Kompetenz bei Agrammatismus an. Als empirische Basis dienen die Resultate aus einer Grammatikalitätsbeurteilung von 451 englischen Sätzen durch vier agrammatische Patienten. Etwa die Hälfte der angebotenen Sätze wiesen grammatikalische Abweichungen unterschiedlichen Typs auf. Die Patienten schnitten bei der Beurteilung der Sätze mit wenigen Ausnahmen gut ab. Interessanterweise gibt es bei den verwendeten Sätzen linguistische Muster, die die Ergebnisse völlig anders erklären könnten. Wie Bayer \& de Bleser (in Vorbereitung) zeigen, können die meisten der von Linebarger et al. verwendeten Grammatikalitätsverletzungen mit einem intakten Lexikon und den darin verzeichneten syntaktischen/semantischen Eigenschaften von Wörtern entdeckt werden. Die Ausnahmen, bei denen die Beurteilungen instabil waren, sind exakt durch solche Strukturen hervorgerufen, bei denen die filternde Wirkung des Lexikons und einer strikt lokalen Syntax versagt, nämlich bei der Analyse von Sätzen, bei denen ,lange“ 
Bindungsrelationen überprüft werden müssen, z. B. [The little boy $]_{\mathrm{i}}$ fell down, didn't he ${ }_{\mathrm{i}} /{ }^{*} i t_{i}$ ?

Ein anderes Problem mit dem Erklärungsansatz von Linebarger et al. ist, daß indirekte Voraussagen gemacht werden, die nicht zutreffen. Wenn es zuträfe, daß bei Agrammatikern semantisches Verarbeiten einen inhibierenden Effekt auf die Syntax hat, so sollte sich die syntaktische Leistung verbessern, wenn die Situation keinen starken Einsatz des semantischen Systems verlangt, z. B. bei formulaischer Sprache, beim Nachsprechen usw. Klinische Erfahrungen mit agrammatischen Patienten sprechen gegen diese Erwartung. Die Patienten sprechen in etwa immer gleich unflüssig und fragmentarisch.

\subsection{Adaptation}

In einem gewissen Sinn eine Verarbeitungsstörung ist Agrarnmatisınus auch nach Ansicht von Heeschen und Kolk ${ }^{12}$. Danach lassen sich Agrammatiker norınalerweise, d.h. in unverstellten Gesprächssituationen nicht auf Strukturen ein, die sie linguistisch nicht bewältigen können. Der Patient paßst sich seiner (irgendwie gearteten) Störung an. Die gestörte (Spontan)-Sprache zeigt demnach das Problem nicht direkt. sondern nur eine Reaktion auf das Problem. Was das zugrundeliegende Problem ist, wird nicht genau gesagt. Die Moglichkeiten reichen von einem partiellen syntaktischen Defizit bis hin zu Störungen des sprach. lichen Gedächtnisses oder der Integration verschiedener linguistischer Aktivitäten. die nötig sind, um eine Proposition zu grammatikalisieren. In diesem Sinn ist die Adaptationstheorie ziemlich vage. Präzisere Voraussagen macht sie hinsichtlich der Entstehung des Agrammatisnus. Nach Heeschens Meinung ist unmittelbar nach Eintritt einer kortikal bedingten Sprachstörung niemals ein Agrammatismus zu erwarten, da dieser erst ,erlernt" werden muk. ${ }^{13}$ Der Patient hat verschiedene Möglichkeiten, auf seine Störung zu reagieren. Er kann sich darüber hinwegsetzen und als Konsequenz Fehler produzieren. Dies ergibt dann die Symptome des Paragrammatismus. Er kann sich aber auch der Störung anpassen und nur das produ. zieren, was ohne zuviel Sprachanstrengung fehlerfrei produziert werden kann. Dies ergibt die Symptome des Agrammatismus. Nach Kolk \& van Grunsven (1985) gibt es zunindest zwei Sorten von Adaptation, die ,korrektive“ und die „präventive“. Diese Differenzierung soll hier aber nicht weiter besprochen werden.

Einwände gegen die Adaptationstheorie liegen auf der Hand. Zunächst ist sie unpräzise hinsichtlich ihrer Annahmen über die Grundstörung. Wichtiger ist aber. daß eine Entscheidungsmöglichkeit des Patienten, so oder so zu sprechen, ziemlich: unplausibel erscheint. Ganz offensichtlich bestehen Schwierigkeiten, zu erklären. warum manche Patienten schon kurz nach Eintritt der Erkrankung ein hartnäckiges agrammatisches Störungsbild zeigen, während andere hartnäckig paragrammatisut: sprechen, d.h. warum es so gut wie nie Übergänge von einem in das andere St 1 rungsbild gibt. Heeschen will das mit einem externen Faktor erklären, nämlich dem: der Depression. Einige Patienten reagieren auf ihre Störung durch Adaptation, wer sie -- bedingt durch ihre Nicht-Flüssigkeit und die oft mit der Erkrankung einher- 
gehenden Lähmung - depressiv sind. Da diese Erklärung zur Zeit nur als unbestätigte Hypothese existiert, wollen wir nicht weiter darauf eingehen.

\subsection{Selektive Syntaxstörungen}

Neuere Forschungen haben sich immer wieder darauf konzentriert zu zeigen, daß Agrammatiker in geeigneten experimentellen Situationen mehr syntaktische Fähigkeiten demonstrieren als ihre spontane Sprachproduktion vermuten lassen würde. Resultate dieser Art sind von besonderem Interesse, als sie nahelegen, daß über gewisse linguistische Strukturen Intuitionen verfügbar sind, aber über andere nicht. Vor allem die Forschungen von Caplan und Grodzinsky halten sich eng an den strukturellen Faktor, den die linguistische Theorie unabhängig zur Verfügung stellt. Da die moderne Syntaxtheorie nach Chomsky (1981) mit verschiedenen Repräsenationsebenen und Subsystemen (linguistischen „,Modulen“) arbeitet, könnte es sein, daß die getroffenen Distiktionen eine Reflexion im Sprachverwendungsprozeß haben und somit auch verschiedene Bereiche gestört sein könnten, ohne daß gleich das gesamte linguistische oder syntaktische Wissen ausradiert ist. Wie wir in der Einleitung gesagt haben, ist es möglich, daß Agrammatiker pränominale Adjektive korrekt nach Genus und Numerus des Nonıens flektieren. Dazu braucht man Syntax, wenn auch nur eine minimale, die sagt, was der Kopf der Konstruktion ist und wie die morphologischen Merkmale weitergegeben werden etc. Das heißt aber mit Sicherheit nicht, daß notwendigerweise auch Bindungs- und Kontrollrelationen beherrscht werden. Mit anderen Worten, der in neuropsychologischen Kreisen häufig benützte Begriff ,Syntax“" ist $u$.U. viel zu naiv, um damit fruchtbar arbeiten zu können. „Syntax“ besteht, wenn man der Argumentation der generativen Grammatik folgt, aus verschiedenen Teilsystemen, die zusammenwirken müssen, um den Sätzen einer Sprache adäquate Strukturbeschreibungen geben zu können. Caplan (1986) nennt mit Recht den Bereich der neurolinguistischen Forschung, der dem durch die Linguistik vorgegebenen strukturellen Faktor eine zentrale Stelle einräumt, ,linguistic aphasiology “, um ihn von anderen Richtungen der Neurolinguistik abzusetzen.

Wir werden im folgenden ausgiebig mit linguistischen Fraktionierungen und deren Bedeutung für die Theorie des Agrammatismus zu tun haben, da wir unsere Arbeit als Teil der linguistischen Aphasiologie sehen. Es wird sich hoffentlich im Verlauf der Untersuchung zeigen, daß dieses Vorgehen fruchtbar ist.

\section{Kasus im Deutschen}

Bevor wir auf das Deutsche eingehen, sind einige einführende Bemerkungen zur Rolle der Kasus allgemein nötig. Zwei Unterscheidungen haben sich theoretisch bewährt: (a) die Unterscheidung von, ,abstraktem " und .,morphologischem “ Kasus, (b) die Unterscheidung von ,lexikalischem" und ..strukturellem" Kasus. 
Wenden wir uns zuerst (a) zu: Das Englische kennt keinen unmittelbar sichtbaren Unterschied zwischen Nominativ und Akkusativ bei NPs wie the dog. Sollte man also argumentieren, daß für diese Sprache die Unterscheidung von Nominativ und Akkusativ irrelevant ist? Wir meinen nein. Kinder, die Englisch erwerben, bekom. men nämlich positive Evidenz für eine solche Unterscheidung durch das Pronominalsystem, das morphologischen Kasus sichtbar bewahrt hat: he/him, she/her. we/us etc. Ist eine solche Erfahrung einmal gemacht, scheint sie sich automatisch auf Kategorien auszuwirken, die distributionell mit der Quelle der Erfahrung verbunden sind: Bill saw him/Bill saw the dog. Dies erklärt, warum Sprecher des Englischen selbst bei NPs wie the dog Kasusintuitionen haben. Es erklärt auch. warum sie - anders als Sprecher des Deutschen - keine vergleichbaren Intuitionen über den Unterschied von Dativ und Akkusativ haben. Es gibt für den Sprachlerner einfach keine Evidenz: Das was im Deutschen Dativ und Akkusativ ist, ist im Englischen ein einziger Kasus, den man mit „,Objektiv“ bezeichnen könnte ${ }^{14}$. Damit ist die Unterscheidung von abstraktem und morphologischem Kasus hergestellt, die sich so zusammenfassen läßt: Ist in einer Sprache einmal ein morphologischer Kasus $\mathrm{K}_{\mathrm{m}}$ (durch positive Evidenz) induziert, so entsteht ein von $\mathrm{K}_{\mathrm{m}}$ unabhängiger, lediglich funktional verbundener, abstrakter Kasus $\mathrm{K}_{\mathrm{a}}$. Morphologischer Kasus ist also der Auslöser, der zur Etablierung eines abstrakten Systems führt.

Wenden wir uns nun (b) zu: Die Unterscheidung von lexikalischein und strukturellem Kasus ist dadurch nahegelegt, daß bestimmte Kasus arbiträr mit bestimmten lexikalischen Kasuszuweisern verbunden sind, während andere von der strukturellen Umgebung in einem Baumgraphen bestimmt sind. Es scheint eine Idiosynkrasie zu sein, daß etwa helfen den Dativ regiert, während gedenken den Genitiv regiert. Andere lexikalische Kasuszuweiser sind im Deutschen Präpositionen und Adjektive. Es ist unklar, ob man den vom Verb regierten Akkusativ als lexikalischen Kasus auffassen soll. Haider (1985) bezeichnet als strukturelle Kasus diejenigen Fälle, die Kasusalternation aufweisen ${ }^{15}$. Demzufolge wäre der vom Verb regierte Akkusativ ein struktureller Kasus. Klare Fälle sind der Nominativ und der Genitiv, wenn letzterer in Spezifizierer- oder in post-NP-Position auftritt.

Nur der Vollständigkeit halber sei hier gesagt, daß das Kasusparadigma des Deutschen, das vier Kasus unterscheidet, defektiv ist. Nur im Maskulin/Singular korrespondieren die vier abstrakten Kasus mit vier unterschiedlichen morphologischen Kasus. Dies wird eine Rolle in den später vorgestellten Experimenten spielen. Dak Sätze wie Das Kind füttert die Frau; Die Kugeln bewegen die Platten; Karl ärgert sich, weil die Dienerin der Königin hilft bei nur geringem Nachdenken als potentiell ambig empfunden werden, ist auf nichts anderes zurückzuführen als auf die oben angesprochene Unabhängigkeit der abstrakten Kasus.

Es ist plausibel, einen Zusammenhang zwischen relativ freier Wortstellung und einem expliziten, d.h. morphologisch verwurzelten Kasussystem zu vermuten. Konzentrieren wir uns zunächst auf morphologisch unterschiedene NPs und die Numeruskongruenz zwischen Subjekts-NP und finitem Verb. Was muß ein syntaktischer Parser leisten, der einen einfachen transitiven Hauptsatz des Deutschen 
analysiert? Unserer Auffassung nach ist das Vorfeld im Deutschen mit geringen Ausnahmen transformationell besetzt. ${ }^{16}$ Es ist unklar, ob der Parser die Vorfeld. Kategorie immer auf eine ( $\mathrm{A}^{\prime}$-gebundene) Spur im Mittelfeld bezieht. Ebenso ist es nicht unmittelbar einsichtig, ob der Parser das in Zweitposition befindliche finite Verb auf die Satzendposition beziehen muß, was sich in der Syntax unabhängig von Parsing-Überlegungen, als die überzeugendste Lösung herausgestellt hat ${ }^{17}$. Klar erscheint jedoch, daß eine NP, die vom Verb regiert wird, auf eine Leerstelle im Mittelfeld bezogen werden muß, wenn sie im Vorfeld auftritt ${ }^{18}$, also

[Den Mann $]_{i}$ hat der Hund $e_{i}$ gebissen

Wir können davon ausgehen, daß der Parser bei Eintritt der NP den Mann eine Suchprozedur einleitet, in der eine Lücke gefunden werden soll, in der die NP abgelegt werden kann. Bei Eintritt des finiten Verbs hat ist klar, daß eine Nominativ-NP im Singular gefunden werden muß, da ja die initiale NP in (6) eine Akkusativ-NP war, die nicht vom Tempusmerkmal des Verbs regiert sein kann.

Was passiert, wenn ein Satz wie (7) verarbeitet werden soll?

\section{Das Kind hat die Oma gefuittert.}

Wie wir oben schon gesagt haben, sind solche semantisch reversiblen Sätze potentiell ambig. Es kommt dabei auf die Qualifizierung, ,potentiell “ an. Da alle Sprecher des Deutschen zunächst an die Interpretation denken, nach der die NP das Kind die semantische Rolle AGENS und die NP die Oma die Rolle PATIENS bekommt, also in Kasusrelationen ausgedrückt NOMINATIV < AKKUSATIV, ist es unwahrscheinlich, daß der Parser eine Analyse wie in (6) überhaupt beschreitet. Im Repräsentationssystem steckt also mehr als das, was der Parser in einem gegebenen Fall tun wird. Das schließt natürlich nicht aus, daß der Parser in gewissen z. B. kontextuell, prosodisch oder durch semantische Selektion bedingten Fälle exakt die in (6) angedeutete Struktur verarbeiten muß.

Es sollte damit klar sein, daß die morphologische Kennzeichnung von Konstituenten durch Kasussuffixe bzw. durch Numeruskongruenz dem Parser hilft, bei zeitgebundener Verarbeitung bereits sehr früh eine Hypothese zu bilden, in der Spurenbindung auftritt. ${ }^{19}$

\section{$4 \quad$ Versuchspersonen}

Im folgenden werden die aphasischen Patienten vorgestellt, die an der Untersuchung teilgenommen haben. Da es sich dabei nicht um eine Gruppenstudie handelt und da die Patienten durchaus nicht immer exakt vergleichbar sind, ist es nötig, jeden Fall genügend genau vorzustellen. Berichtet werden sollen fünf Versuchspersonen, von denen drei (nämlich C.B., H.R. und M.H.) Agrammatiker sind und zwei (P.P. und H.H.) Paragrammatiker. Obwohl es primär um Agrammatismus geht, ist es interessant, das Verhalten paragrammatischer Patienten im Vergleich zu 
sehen, vor allem deshalb. weil sowohl in der aphasiologischen Tradition als auch in neuerer Zeit wieder dafür argumentiert wurde, die beiden Störungstypen auf dieselbe Grundstörung zurückzuführen ${ }^{20}$.

\subsection{C.B.}

C.B. war ein 72jähriger gelernter Bäcker, der ca. 28 Jahre vor der Untässlchung einen linkshirnigen Insult erlitten hat, der einen sehr weiträumigen Substan . defekt auslöste, wobei das Versorgungsgebiet sämtlicher Mediaäste mit Ausnah!ne der hinteren Temporalarterie betroffen war. C.B.'s Lähmung hatte sich so gut wie vollständig zurückgebildet. Der Patient sprach kontrolliert und konzentriert. he: nur etwas verlangsamtem wortinternen Sprechtempo. Offenbar lagen schwcro Wortfindungsstörungen vor. C.B.'s Sprachverständnis schien gut. Das herausragen de Kennzeichen war C.B.'s Agrammatisnuus. Hier ist ein Auszug aus einem Interview mit C.B. in Rahmen einer klinischen Untersuchung:

Frage: Was haben Sie denn im Urlaub gemacht?

C.B.: ... lang schlafen und äh ... Kaffee getrunken ...

spazieren gehen. und dann. essen. und äh Stunde niedergelegt. dann

Kaffee getrunken.

Bei der Beschreibung von Situationsbildern, die Teil des AAT ist, produzierte C.B. etwas reichere Syntagmen, -- ein Phänomen, das nicht nur bei diesem Patienten anzutreffen ist.

a) Ziel: Der Junge weint, weil er ein Glas zerbrochen hat.

Reaktion: Das ist äh äh der zerbrochene Krug oder was und äh äh ... Kind älı weint

b) Ziel: Der Mann liegt auf der Couch, raucht Pfeife und liest die Zeitung.

Reaktion: Pfei ... der Mann raucht ... die Zeitung lesen

C.B. hatte eine einfache Schulbildung und sprach prämorbid rheinische Umgangssprache vom Typ Kölsch. Im AAT wurde ein klarer Fall von Broca-Aphasie diagnostiziert.

\subsection{H.}

M.H. war eine 54jährige Aachener Hausfrau, die ca. 9 Jahre vor der Untersuchung einen linkshirnigen Insult erlitten hatte, ebenfalls mit einer sehr ausgedehnten Läsion im gesamten Mediabereich. Bei M.H. betand initial wohl eine globale Aphasie, die sich aber im Laufe der Zeit zurückbildete. Im Gegensatz zu C. B. sprach M.H. fluissiger, dafür aber mit reichlich sprachlichen Stereotypien wie ich weiß nicht oder Floskeln wie oh Gott. Wir baten M.H. die Geschichte von Rotkäppchen zu erzählen. Hier ein Auszug: 
(10) Der Wolf hat das Rotkäppchen ... gesucht und ... äh ... Wald. Wald und die Flasche ... und ... äh ... ja und äh wuh-wuh und der der Wolf Großmutter gut ... nein gefressen und ... äh Häubchen da ... äh ... das Häubchen.und. äh das . äh und Reg ... äh Rotkäppchen ... äh schlimm das Rotkäppchen ... frißt auf ... auf ... und der Jäger

In (11) werden Beispiele von M.H.'s Beschreibung von Situationsbildern gegeben:
a) Ziel: Der Mann bettelt
Reaktion: Der ... Mann ... Bettler
b) Ziel: Die beiden Männer schreien sich an
Reaktion: Der Mann . und. der Mann . schreit sich an

M.H. hatte in ihrer Jugend als Sekretärin gearbeitet, hatte aber mit Sicherheit eine einfache Schulbildung. Ihre Sprache war stark dialektgefärbt (Aachenerisch). Im AAT wurde eine Broca-Aphasie diagnostiziert.

\subsection{H.R.}

H.R. war eine 47jährige ehemalige Chefsekretärin bei einem großsen Industrieunternehmen. Sie hatte zum Zeitpunkt der Untersuchung eine 25 Monate alte Aphasie, die ebenfalls auf einen linkshirnigen Insult zurückzuführen ist, sowie eine rechtsseitige Lähmung. H.R. hatte eine weniger grokse, elıer frontal gelegene Läsion. Ihre Spontansprache war ziemlich flüssig und sicherlich besser als die von C.B. und M.H. Dennoch bestand ein deutlicher Agrammatismus, wie der Auszug aus einem Interview in (12) zeigt:

(12) Frage: Was machen Sie an einem normalen Werktag?

H.R.: Ja ich. stehe auf um neun Uhr. ungefähr und. dann wasche mich oder. ja. und. ziehe um. ziehe ich. ziehe ich dann üh sehe ich das Was. sehe ich das Wetter. dann äh ich äh brummig und gehe hinunter im Wohnzimmer und. trinke Kaffee. mit Papa und essen so. Brot oder Brötchen.

H.R. beschrieb Bilder wie folgt:
a) Ziel: $\quad$ (siehe 9a)
Reaktion: Das Kind weint. die Vase ist kaputt
b) Ziel: (siehe 9b)
Reaktion: Die La. der Mann liegt auf dem Sofa und liest die Zeitung

Anders als C.B. und M.H. hatte H.R. eine gehobene Schulbildung und war keine Dialektsprecherin. Ihre Tätigkeit als Chefsekretärin legt nahe, daß sie prämorbid sehr viel mit Sprache zu tun hatte. Auch hier wurde eine Broca-Aphasie diagnostiziert. 


\section{$4.4 \quad$ P.P.}

P.P. war ein 4 ljähriger Elektroingenieur, der sehr viel im Ausland zu tun hatte. P.P. hatte 21 Monate vor der Untersuchung einen linkshirnigen Insult erlitten, der temporo-parieto-occipitales kortikales Gewebe zerstörte. Die flüssige Spontansprache war initial vom Typ eines semantischen Jargons, besserte sich aber bis zum Zeitpunkt der Untersuchung nicht unerheblich, obwohl ein Paragrammatisınus zurückblieb. P.P. hatte von Anfang an ein relativ gut erlaltenes Sprachverständnis, das zum Untersuchungszeitpunkt laut Test unbeeinträchtigt war. ${ }^{21}$ (14) gibt einen Auszug aus einem Routineinterview mit P.P.:

(14) Frage: Haben Sie noch Kontakt mit früheren Kollegen?

$P . P .:$ Nein ich bin jetzt praktisch sozusagen drei Jahre für Urlaub beurlaubt. für drei Jahre. natürlich in Elektro ist das sehr schwer wenn man da bleibt zu einem Zeitpunkt praktisch man muß auch lernen und das kann ich nicht mehr und das wird jetzt bitter für mich. Wenn ich jetzt in eine neue Sache reinkomme und dann da krieg ich jetzt nicht erstmal klar was es war. das muß man auch aufwisch auf aufarbeiten oder auf. das vergißt man immer.

P.P. beschrieb Situationsbilder wie folgt:
a) Ziel: Vater und Sohn spielen zusammen Indianer.
Reaktion: Der Vater spielt mit Sohn. was spielt sie? Indianer spielt.
b) Ziel: Der Polizist verhaftet den Dieb
Reaktion: Polizist stellt die Schelle ein Arresti. Arresten...
c) Ziel: $\quad$ Die Lehrerin erklärt dem Kind etwas an der Tafel
Reaktion: Lehrerin schreibt an der Tafel und steht davor ein Kind.

P.P. hatte ebenfalls eine gehobene Schulbildung und sprach kaum Dialekt. Bedingt durch seinen Beruf hatte er auch Fremdsprachenkenntnisse, die aber nicht systematisch überprüft wurden. P.P. hatte im AAT herausragende Störungen beim Nachsprechen. Es wurde eine Leitungsaphasie diagnostiziert.

\subsection{H.H.}

H.H. war ein 33jähriger selbständiger Floristenmeister, der ca. 5 Jahre vor der Untersuchung einen linkshirnigen Insult erlitten hatte. H.H. mußte sich daraufhin einer Operation an der Carotis unterziehen. Im CT stellte sich ein ausgedehnter Mediainfarkt links dar.

H.H. sprach paragrammatisch, was aber eher durch viele Satzabbrüche bedingt schien. In allen durch den AAT erfaßten Bereichen zeigten sich leichte Störungen. Ebenso wie bei P.P. war jedoch das Nachsprechen herausragend gestört.

(16) gibt ein Beispiel der Spontansprache von H.H.; der Patient berichtet dabei über den Beginn seiner Sprachstörung: 
... und da war meine Schwester M. hier . die war . halb acht zum Frühstück hier .. mit dem Sprechen war gar nichts hier .. kann mir (nimmer) .. tja dann bin ich .. wieder Treppe hoch. Schlafzimmer hinger ... liegt ich im Bett hier . dann hab ich .. so zwei Wochen bin ich bin ich im Bett geschlafen . Krankenhaus .. bin ich . ja Warstein im kleinen Krankenhaus .. und die Ärzte haben für mich nicht hier .. ne waschen... ne Essen hier . dann . ja .. und dann .. gar nach . ja vor wordsen . und dann bin ich dann bin ich essen (oder „Essen“) hier . also vier Wochen bin ich im Krankenhaus hier

H.H. beschrieb Situationsbilder wie in (17) gezeigt:
a) Ziel: Die Frau putzt die Kanne.

Reaktion: Die Mutter spült . spült . mit . mit die . Kanne.

b) Ziel: Der Mann hat einen Stiefel geangelt.

Reaktion: Der Mann sitzt auf . äh in dem äh in dem Bo . Boot und und hebt .. hiebt . mit den . mit dem Angel.

c) Ziel: $\quad$ Die beiden streiten sich.

Reaktion: Die zwei Männer .. quatschen oder haben ganz laut ... sprechen.

H.H. hatte mehrere Fachschulen besucht und sich in verschiedenen Städten des Landes aus Gründen der Ausbildung aufgehalten. Seine Bildung kann als durchschnittlich angesehen werden.

In der AAT-Untersuchung wurde eine Leitungsaphasie diagnostiziert.

\section{$5 \quad$ Experimente}

Im folgenden stellen wir die Kasusexperimente vor, die mit den in 4 beschriebenen Patienten sowie mit einer Gruppe von 15 neurologisch unauffälligen Kontrollpersonen durchgeführt wurden. Die Kontrollpersonen wurden dabei so ausgewählt, daß sie hinsichtlich Alter und Bildung mit den Patienten vergleichbar waren. Sie stammten fast durchgehend, so wie die Patienten, aus der Umgebung von Aachen und der Düsseldorfer Gegend. Der Altersbereich ging von 20 bis 72 Jahren. Das mittlere Alter lag bei 52.3 Jahren. Die Kontrollpersonen wurden post hoc in zwei Gruppen eingeteilt, die der Einfachheit ,gute Normale " und ,schlechte Normale" genannt werden sollen.

Alle fünf schlechten Normalen hatten eine nur einfache Bildung und verwendeten in ihrer stark dialektal gefärbten Umgangssprache das Kasussystem des Standarddeutschen kaum. Es sei darauf hingewiesen, daß die rheinische Umgangssprache und das Aachenerische bezüglich des Kasussystems eher dem Niederländischen als dem Deutschen entspricht. So gibt es z.B. keinen Genitiv, sondern lediglich die bekannten periphrastischen Konstruktionen mit Possessivpronomen, z. B. dem Vater sein Haus oder mit einer von-PP wie das Haus vom Vater. Soweit wir es beurteilen können, werden nur Pronominalformen bezüglich Kasus morphologisch unterschieden, nicht aber volle NPs. ${ }^{22}$ 
Die zehn guten Normalen hatten überwiegend eine mittlere Bildung, in zwei Fällen allerdings auch eine einfachere Bildung. Ihre Umgangssprache war durchgehend weniger dialektgefärbt.

\subsection{Experiment I: kanonische und nicht-kanonische Wortfolge}

In diesem Experiment wurden die Versuchspersonen gebeten, Sätze vervollständigend zu lesen, in denen eine NP, bestehend aus Art + Adj + N unflektiert war. Es kam dabei auf drei Aspekte an: Erstens stellte sich die Frage, ob Aphasiepatienten überhaupt die Fähigkeit besitzen, so wie Heeschen vermutete, solche NPs intern korrekt zu flektieren: zweitens, ob sie auch die syntaktische Rolle der (morphologischen) Kasus ausnützen können und drittens, falls ja, ob sie dazu auch in der Lage sind, wenn von der ,,kanonischen“ Konstituentenfolge SVO abgewichen wird.

\subsubsection{Material}

Den Patienten wurden Sätze der folgenden Art vorgegeben.

\section{kanonisch}

NOM: Ein ... hungrig ... Bettler trifft einen reichen Mann

AKK: Ein fähiger Seeman braucht ein ... streng ... Kapitän

DAT: Ein alter Gärtner droht ein ... jung ... Koch nicht-kanonisch

Einen hungrigen Bettler trifft ein ... reich ... Mann

Ein ... fähig ... Seemann

braucht ein strenger Kapitän

Ein ... alt ... Gärtner droht

ein junger Koch

Es wurden insgesamt 60 Sätze vorgegeben. Jeder Kasus war also 20 mal zu ergänzen, - davon 10mal in kanonischer und $10 \mathrm{mal}$ in nicht-kanonischer Position, wenn man davon ausgeht, daß der Nominativ im Vorfeld die unmarkierte Form darstellt.

\subsubsection{Durchführung}

Alle Versuchspersonen, also auch die Kontrollpersonen, wurden individuell getestet, nachdem die Aufgabe erklärt und an zwei Übungsbeispielen erläutert war. Die verschiedenen in (18) gezeigten Strukturtypen wurden randomisiert angeboten, um Set-Effekte zu vermeiden. Die Versuchsperson konnte immer nur den gerade zu bearbeitenden Satz sehen. Bei den Patienten wurde entweder eine Tonbandaufzeichnung der Untersuchung gemacht oder aber es wurde (bei sehr langsamen Patienten) mitprotokolliert. Patienten, die Schwierigkeiten beim Lesen hatten, wurde vom Untersucher in jeder Hinsicht geholfen, sofern eine solche Hilfe die Lösung der Aufgabe nicht berührte. 


\subsubsection{Ergebnisse}

Die Ergebnisse für die guten Normalen sind in Tabelle 1, die für die schlechten Normalen in Tabelle 2 gegeben. Bei diesen Gruppen sind immer Mittelwerte in Prozent angegeben. Der Übersichtlichkeit halber sind die korrekten Ergebnisse eingerahmt.

Schwierigkeiten bei der Kasuszuweisung in eine nicht-kanonische Position zeigen sich bereits bei den guten Normalen, wo z. B. $13 \%$ der präverbal einzusetzenden Akkusativformen durch Nominativformen ersetzt werden. Bei den schlechten Normalen verstärkt sich dieser Effekt dramatisch. Da hier bereits in der kanonischen Bedingung auffallend viele Ersetzungen beim Dativ auftreten, kann man davon ausgehen, daß in dieser Gruppe sehr schlechte Intuitionen über den Gebrauch des Dativs allgemein bestehen.

Da es zu erwarten war, daß aphasische Patienten bereits mit der NP-internen Flexion Probleme haben, war es ratsam, weitere Auswertungskategorien für die Daten bereitzustellen. Wir haben uns dazu entschieden, intern falsch flektierte NPs in zwei Kategorien einzuteilen, nämlich solche, die bei gutwilliger Interpreta-

Tabelle 1 Ergebnisse für die ,guten Normalen“ ( $(n=10)$

\begin{tabular}{|c|c|c|c|c|}
\hline & & \multicolumn{3}{|c|}{ REAKTION } \\
\hline \multirow{4}{*}{ 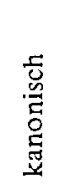 } & ZIEL & NOM & $\mathrm{AKK}$ & DAT \\
\hline & NOM & 100 & 2 & 2 \\
\hline & $\mathrm{AKK}$ & -- & 98 & -- \\
\hline & DAT & -- & -- & 98 \\
\hline \multirow{3}{*}{ 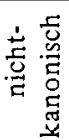 } & NOM & 97 & 13 & 2 \\
\hline & AKK & 3 & 85 & 1 \\
\hline & DAT & -- & 2 & 97 \\
\hline
\end{tabular}

Tabelle 2 Ergebnisse für die ,schlechten Normalen“ ( $n=5)$

\begin{tabular}{|c|c|c|c|c|}
\hline & & \multicolumn{3}{|c|}{ REAKTION } \\
\hline \multirow{4}{*}{ 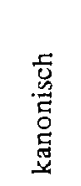 } & ZIEL & NOM & AKK & DAT \\
\hline & NOM & 76 & 2 & 8 \\
\hline & $\mathrm{AKK}$ & 20 & 98 & 56 \\
\hline & DAT & 4 & -- & 36 \\
\hline \multirow{3}{*}{ 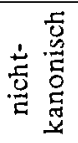 } & NOM & 50 & 30 & 58 \\
\hline & $\mathrm{AKK}$ & 46 & 70 & 30 \\
\hline & DAT & 4 & - & 12 \\
\hline
\end{tabular}


Tabelle 3 Ergebnisse für die Agrammatiker (C.B., M.H., H.R.)

\begin{tabular}{|c|c|c|c|c|c|c|c|c|c|c|c|c|c|c|c|c|}
\hline \multirow{6}{*}{ 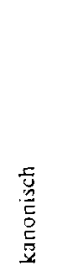 } & \multirow{3}{*}{ ZIEL } & \multicolumn{15}{|c|}{ REAKTION } \\
\hline & & \multicolumn{3}{|c|}{ NOM } & \multicolumn{3}{|c|}{ AKK } & \multicolumn{3}{|c|}{ DAT } & \multicolumn{3}{|c|}{$\begin{array}{l}\text { defektive } \\
\text { Kongruenz }\end{array}$} & \multicolumn{3}{|c|}{ uninterpretierbar } \\
\hline & & C.B. & M.H. & H.R. & C.B. & M.H. & II.R. & C.B. & M.J!. & II.R. & C.B. & M.II. & H.R. & С.в. & M.H. & H.R. \\
\hline & NOM & -- & 80 & 100 & 100 & 20 & -- & -- & $\ldots$ & -- & 40 & -- & $-\cdots$ & $\cdots$ & - & -- \\
\hline & $\mathrm{AKK}$ & -- & 70 & $-\cdots$ & 100 & 30 & 70 & $=-$ & $-\cdots$ & 30 & 20 & -- & 10 & -- & -- & -- \\
\hline & $\mathrm{D} \wedge \mathrm{T}$ & -- & 30 & 10 & 90 & 50 & 50 & 10 & 10 & 20 & 10 & 10 & 10 & -- & 10 & 20 \\
\hline 5 & NOM & -- & 50 & 10 & 100 & 40 & 50 & -- & $\cdots-$ & 40 & -- & 10 & -- & -- & 10 & $-\cdot \cdot$ \\
\hline 穵光 & $\mathrm{AKK}$ & $\cdots$ & 70 & 100 & 100 & 30 & -- & -- & -- & -- & 10 & --- & -- & -- & $\cdots$ & -- \\
\hline E & DAT & 20 & 70 & 90 & 60 & 30 & -- & 20 & -- & $\ldots$ & 30 & -- & -- & -- & $-\cdots$ & 10 \\
\hline
\end{tabular}

tion noch einem Kasus zugerechnet werden konnen und solche, die keine eindeutige Interpretation erlauben. Defizitäre Kongruenz liegt z. B. vor in *ein kleinen Fuchs statt einen kleinen Fuchs; Nichtinterpretierbarkeit hingegen liegt vor in Beispielen wie *einen kleiner Fuchs oder in Fällen die auch Genusfehler einschließen, wie *ein kleines Fuchs. Die Reaktionen mit defizitärer Kongruenz wurden in die Auswertung bezüglich Kasus einbezogen.

Die Ergebnisse für die Agrammatiker C.B., M.H. und H.R. sind in Tabelle 3 getrennt in Prozentzahlen aufgeführt.

Dabei fällt zunächst auf, daß es kaum zu uninterpretierbaren Reaktionen kommt; auch die Fälle mit defektiver Kongruenz halten sich in Grenzen. Andererseits produzieren alle drei Patienten, wenn auch in unterschiedlichem Maß alle Ziel-Kasusformen. Wie nicht anders zu erwarten, ist dabei der Dativ am schwächsten repräsentiert. Hingegen scheint der Akkusativ vielfach der ,Kasus des Versagens“ zu sein. Besonders die Ergebnisse von C.B. legen nahe, daß der Patient den Akkusativ perseveriert hat. M.H. dagegen scheint als, default'-Form, wenn auch nicht so stark, den Nominativ zu benützen. In der kanonischen Bedingung zeigt H.R. am ehesten linguistisch sinnvolle Reaktionen. Allerdings hält sie an ihrer Strategie auch in der nicht-kanonischen Bedingung fest. Dieses Festhalten an einer SVO-Konstituentenfolge bewirkt, daß H.R. - ähnlich wie die schlechten Normalen - im Vorfeld von OVS-Sätzen nur Nominative produziert, während sie in die postverbale Position fast nur Akkusativ- und Dativformen einsetzt.

Tabelle 4 gibt die Resultate für die beiden paragrammatischen Patienten P.P. und H.H. wieder.

Im Gegensatz zu den Agrammatikern häufen sich hier die Reaktionen mit defektiver Kongruenz. Im wesentlichen lagen bei beiden Patienten Formen vor, bei denen die Flexion am Artikel ausgelassen war, z. B. *ein fleißigen Fahrer. Im Gegensatz zu P.P. zeigte H.H. starke Unsicherheiten mit dem Genus, was den Ausschlag 
Tabelle 4 Ergebnisse für die Paragrammatiker (P.P., H.H.)

\begin{tabular}{|c|c|c|c|c|c|c|c|c|c|c|c|}
\hline \multirow{6}{*}{ 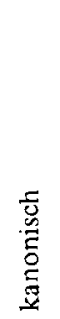 } & \multirow{3}{*}{ ZIEL } & \multicolumn{10}{|c|}{ REAKTION } \\
\hline & & \multicolumn{2}{|c|}{ NOM } & \multicolumn{2}{|c|}{$\mathrm{AKK}$} & \multicolumn{2}{|c|}{ DAT } & \multicolumn{2}{|c|}{$\begin{array}{l}\text { defektive } \\
\text { Kongruenz }\end{array}$} & \multicolumn{2}{|c|}{$\begin{array}{l}\text { uninter- } \\
\text { pretierbar }\end{array}$} \\
\hline & & & H.H. & P.P. & H.H. & P.P. & H.H. & P.P & H.H. & P.P. & H.H. \\
\hline & NOM & 20 & 20 & 60 & 60 & 20 & -- & $\ldots$ & 60 & -- & 20 \\
\hline & $\mathrm{AKK}$ & & 10 & 100 & 40 & $\ldots$ & -- & 10 & 20 & --- & 50 \\
\hline & DAT & 10 & 10 & 70 & 60 & 20 & $\ldots$ & 10 & 20 & -- & 30 \\
\hline \multirow{3}{*}{ 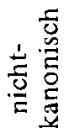 } & NOM & $\cdots$ & 20 & 90 & 60 & 10 & ..... & 20 & 10 & -- & 20 \\
\hline & AKK & 10 & 10 & 90 & 70 & -- & -- & 30 & 50 & $\ldots$ & 20 \\
\hline & DAT & 20 & 40 & & 50 & -- & $\overline{---}$ & 10 & 40 & -- & 10 \\
\hline
\end{tabular}

gegeben haben könnte, daß viele Reaktionen in bezug auf Kasus uninterpretierbar waren. H.H. produzierte Beispiele wie in (19):
a) (... begegnet) einen fähige Arzt
b) Einen brave Küster (lobt ...)
c) Eine gelben Ball (berührt ...)
d) (... schlägt) eine tapfere Ritter
e) (... droht) eine junge, jungen, junge, junges Koch

Ähnlich wie C.B. tendierte P.P. dazu, prinzipiell den Akkusativ einzusetzen. Es versteht sich von selbst, daß solche Strategien (oder zwanghaften Perseverationen) dazu führen, daß auch die tatsächlichen Treffer nicht als strukturelle Leistung bewertet werden können.

Das zweifellos überraschendste an Experiment I ist, daß es Normalpersonen gibt, die sich offenbar in einer experimentellen Situation den Erfordernissen variabler Konstituentenabfolge nicht anzupassen vermögen. Ein starker Einwand gegen das Experiment mit den Aphasikern ist, daß man von Aphasikern nicht mehr erwarten könne als von Normalpersonen. Wir möchten dreierlei dagegenhalten: Erstens bestand immer noch die Frage, wie Agrammatiker (und Paragrammatiker) mit der NP-internen Kongruenz umgehen können; zweitens fallen sowohl H.R. als auch P.P. mit Sicherheit in die soziale Gruppe der ,guten Normalen“. Beide haben eine höhere Schulbildung genossen und haben prämorbid in relativ anspruchsvollen Berufen gearbeitet, in denen es auf sprachliche Differenzierungen durchaus ankommt. Die schlechten Leistungen beider Patienten müssen also auf das Konto der aphasischen Störung gehen; drittens schneiden alle Aphasiker mit Ausnahme von H.R. bereits unter der kanonischen Bedingung schlechter ab als die ,schlechten Normalen", d.h. auch dies geht rein auf die Aphasie zurück.

Es scheint in etwa zwei Wege zu geben, Problemen mit der in Experiment I gestellten Aufgabe zu begegnen: (a) Man läßt sich von der Tatsache, daß manchmal 
präverbal eine nicht-nominativische Form auftaucht, nicht beeindrucken und hält stur an der kanonischen Konstituentenfolge fest, (b) man läßt sich von dieser Tatsache beeinflussen und versucht, das Beobachtete (mit geringen Erfolg) selbst zu reproduzieren, wobei sich dieses Verhalten natürlich mit Strategie (a) mischen kann. Strategie (a) ist durch H.R. (und durch die schlechten Normalen) exemplifiziert, Strategie (b) mehr oder minder durch den Rest der Patienten.

\subsection{Experiment II: Markierte und unmarkierte Abfolge in Doppel- objektkonstruktionen}

Experiment II ist Experiment I bezüglich Versuchspersonen und Art der Durchprüfung gleich, so daß hier sofort auf Materialien und Ergebnisse etc. eingegangen wird.

\subsubsection{Material}

Alle zu vervollständigen Sätze enthielten Doppelobjektverben, die den Objekts-NPs jeweils einen Dativ und einen Akkusativ zuweisen, also Verben wie geben, schenken, widmen, leihen. Da die Klasse von Verben, bei denen zwei Objekte strikt subkategorisiert sind, sehr klein ist, wurden auch Verben hinzugenommen, die optional einen Dativ-Mitspieler zu sich nehmen, z. B. gestehen, rauben, übergeben, waschen. Um die linguistische Kapazität der Patienten auf das Wesentliche zu lenken, wurde auf komplexere NPs verzichtet. Es war nur der definite Artikel in die jeweilige Kasusform zu setzen. Die verwendeten NPs waren durchgehend so, daß nur eine davon, da menschlich oder belebt, die semantische Rolle BENEFAKTIV erhalten konnte. Von insgesamt 30 Sätzen erschienen 20 in der unmarkierten NP-Abfolge Dativ < Akkusativ und 10 in der markierten Abfolge Akkusativ < Dativ. (20) gibt Beispiele des Untersuchungsmaterials, so wie es die Versuchspersonen vorgelegt bekamen:

i. unmarkierte NP-Abfolge

a) Der Minister verleiht d... Sportler d... Orden

b) Der Polizist übergibt d... Anwalt d... Revolver

c) Der Dichter widmet d... Leser d... Roman

ii. markierte Abfolge

a) Der Aufseher schenkt d... Hamster d... Gärtner

b) Der Betrieb schuldet d... Gewinn d... Staat

c) Der Rektor leiht d... Atlas d... Lehrer

\subsubsection{Ergebnisse}

Beide Kontrollgruppen, die Guten wie die Schlechten, hatten bei dieser Aufgabe keine oder nur minimale Schwierigkeiten. 
Tabelle 5 zeigt die Ergebnisse für die Agrammatiker. Die Angaben geben, wie oben, Prozentanteile wieder. Die Spalte rechts außen zeigt summarisch, in wievielen Fällen beide NPs korrekt flektiert wurden.

Die Reaktionen von M.H. sind insofern uninteressant, als die Patientien fast ausschließlich den Akkusativ verwendet. Am besten schneidet H.R. ab, die in der unmarkierten Konstituentenfolge $30 \%$ und in der markierten $40 \%$ der Testsätze korrekt vervollständigt. Bemerkenswerte Fehler in den Reaktionen von H.R., die aus der Tabelle nicht hervorgehen, sind in (21) aufgelistet:

a) Der Polizist übergibt den Anwalt der Revolver

b) Der Dichter widmet der ... den Leser der Roman

c) Der Lehrer verzeiht den Schüler der Ausrutscher

d) Der Dieb raubt den Juwelier dem Schmuck

e) Der Knabe stiehlt den Kutscher dem Hut ... der Hut

Man kann die Fälle $(21 \mathrm{a}-\mathrm{c})$ so interpretieren, daß das Verb die erste NP kasusmarkiert, aber die zweite, ,äußere" NP nicht mehr erreicht. Diese bleibt dann unintegriert und bekommt als ,default"-Wert den Nominativ. Letzteres geschieht eventuell im Sinne einer Benennung, d.h. das Syntagma ist bereits geschlossen, wenn die letzte NP kasusmarkiert werden muß. (21 d, e) sind insofern interessant, als hier die markierte Folge AKK <DAT Testsätze erfaßt, die von der semantischen Struktur her nur die unmarkierte Folge DAT $<$ AKK zulassen. H.R. ist prinzipiell in der Lage, beide Abfolgen zu realisieren. Das geht aus Tabelle 5 hervor. Dennoch gibt es Probleme, diese Fähigkeit mit der jeweiligen semantischen Struktur in Einklang zu bringen. Die folgenden Beispiele von H.R. zeigen, wenn auch nicht so schön, daß auch die markierte Bedingung von der Anordnung DAT $<$ AKK überrollt werden kann:

a) Der Koch borgt dem.das.den.der.das Essig der Kellner

b) Der Rektor leiht dem Atlas dem Lehrer

Die Ergebnisse von C.B. zeigen, daß der Patient über die Form des Dativs verfügt, aber kaum in der Lage ist seine Rolle in ditransitiven Sätzen auszunützen. Die interessanten Fragen bezüglich C.B. sind jedoch, wieso in seinen Reaktionen Genitive auftreten und wieso sie nur in der markierten Bedingung auftreten. Das Rätsel ist schnell gelöst, wenn man sich die jeweiligen Testsätze genauer ansieht. (23) listet C.B.'s Reaktionen auf: ${ }^{23}$

a) Der Aufseher schenkt ein Hamster des Gärtners

b) Der Betrieb schuldet einen Gewinn des Staates

c) Der Schwimmer verdankt ein Sieg des Trainers

d) Der Rektor leiht des.des ... der Atlas des Lehrers

e) Der Wirt verspricht ein Likör des Säufers

In jedem dieser Fälle entsteht eine wohlgeformte syntaktische Substruktur, nämlich:

$$
\left[\mathrm{N}^{\prime \prime} \ldots\left[\mathrm{N}^{\prime} \cdots\left[\mathrm{N}^{\prime \prime} \mathrm{GEN} \cdots\right]\right]\right]
$$


Tabelle 5 Ergebnisse für die Agrammatiker (C.B., M.H., H.R.)

\begin{tabular}{|c|c|c|c|c|c|c|c|c|c|c|c|c|c|c|c|c|c|c|}
\hline \multirow{5}{*}{ 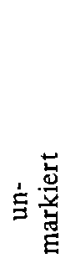 } & \multirow{5}{*}{$\begin{array}{l}\text { ZIEL } \\
\text { 1. NP/DAT } \\
\text { 2. NP/AKK }\end{array}$} & \multicolumn{17}{|c|}{ REAKTION } \\
\hline & & \multicolumn{3}{|c|}{ NOM } & \multicolumn{3}{|c|}{ AKK } & \multicolumn{3}{|c|}{ DAT } & \multicolumn{3}{|c|}{ GEN } & \multicolumn{3}{|c|}{ uninterpretierbar } & \multicolumn{2}{|c|}{ beide NPs korrekt } \\
\hline & & C.B. & M.H. & H.R. & C.B. & M.H. & H.R. & C.B. & M.H. & H.R. & C.B. & M.H. & H.R. & C.B. & M.H. & H.R. & C.B. & M.H. H.R. \\
\hline & & 30 & -- & -- & 45 & 95 & 65 & 15 & -- & 35 & -- & -- & -- & 10 & 5 & -- & 5 & 30 \\
\hline & & 10 & 15 & 20 & 75 & 80 & 70 & -- & $\cdots$ & 10 & -- & -- & $--\cdot$ & 15 & 5 & -- & & \\
\hline \multirow{2}{*}{ 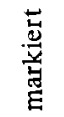 } & 1. NP/AKK & 50 & -- & -- & 30 & 100 & 70 & 10 & -- & 10 & 10 & -- & -- & -- & -- & 20 & \multirow{2}{*}{--} & \multirow{2}{*}{--} \\
\hline & 2. NP/DAT & -- & $-\cdots$ & 10 & -- & 80 & 30 & 20 & -- & 60 & 50 & -- & -- & 30 & 20 & -- & & \\
\hline
\end{tabular}


Tabelle 6 Ergebnisse für die Paragrammatiker (P.P., H.H.)

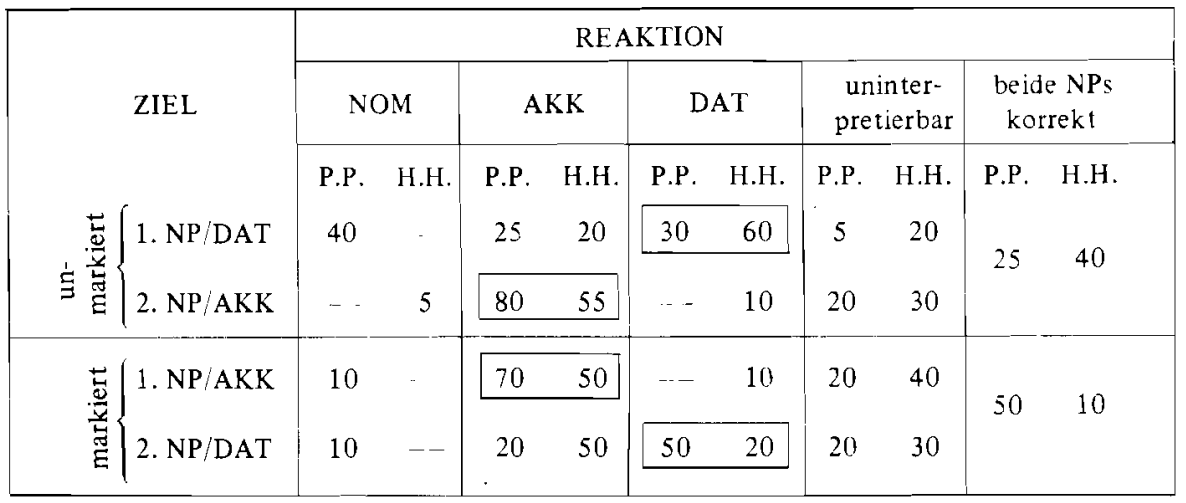

Warum kommt es nie zu Fällen wie der Friseur wäscht den Lehrling des Kopfes, der Minister verleiht den Sportler des Ordens etc.? Die Antwort muß heißen: weil C.B. gezielt semantisch arbeitet. Es ist bezeichnend, daß C.B. in den restlichen Fällen, in denen er keinen Dativ einsetzen konnte, Null-Reaktionen brachte. Wir werden auf diese interessanten Daten zurückkommen.

Die Ergebnisse der paragrammatischen Versuchspersonen (P.P. und H.H.) sind in Tabelle 6 dargestellt.

Wie inan sieht, kommt es zu mehr korrekten Resultaten aber auch zu mehr uninterpretierbaren. Nicht-integrierbare NPs an zweiter Stelle treten kaum auf. Die relativ gut erhaltenen Fähigkeiten, Kasus zuzuweisen, werden häufig überlagert durch falsche Genusselektion und falsche morphologische Kasusrealisation. Beispiele für letzteres finden sich in den Reaktionen von P.P. Wie (25) zeigt, produziert P.P. Augmentivformen, die auf Unsicherheiten im Umgang mit den morphologischen Paradigmata des Deutschen schliefsen lassen:

a) Der Autor widmet denen Krimi denen Sträfling

b) Der Minister verleiht der Sportler denen Orden

c) Der Polizist übergibt den Anwalt desses ... denen Revolver

Andererseits sind die paragrammatischen Patienten in der Lage, diese Schwierigkeiten zu überwinden und sich der Aufgabe anzupassen. Dieser Eindruck entsteht u. a. dadurch, daß sich beide Versuchspersonen im Lauf des Experiments verbesserten. ${ }^{24}$

\subsection{Experiment III: Lexikalischer und struktureller Kasus}

Während die Bedingungen in den Experimenten I und II von den Versuchspersonen die Verarbeitung diskontinuierlicher Abhängigkeiten verlangten, ging es in 
Experiment III darum, die Kasuszuweisung nur in Abhängigkeit von einem adjazenten (lexikalischen oder strukturellen) Kasuszuweiser zu studieren. ${ }^{25}$ Art der Durchführung sowie Versuchspersonen waren wieder wie in den beiden vorangegangenen Untersuchungen. Die Fragestellung war: Wie gehen Aphasiker mit einer analogen Vervollständigungsaufgabe um, wenn nicht-verbale Elemente die Kasuszuweisung steuern, d.h. wenn die Syntagmen besonders klein und nicht abhängig von anderen Elementen im Satz sind?

\subsubsection{Material}

Als lexikalische Kasuszuweiser wurden Präpositionen gewählt, als strukturelle Kasuszuweiser NPs (bzw. je nach Analyse, $N^{\prime}$ ), die einer rechts-adjungierten NP den Genitiv zuweisen. In (26) sind Beispiele aus den Untersuchungsmaterialien angegeben:

i. Präpositionen mit Akkusativ oder Dativ

a) Ein neues Auto fährt gegen ein ... morsch ... Zaun

b) Ein rüstiger Rentner segelt zu ein ... einsam ... Strand

ii. Genitiv in Abhängigkeit von NP (bzw. $\left.N^{\prime}\right)$

a) Der Arbeiter rettet den Sohn ein ... treu ... Kumpel ...

b) Die Frau ein ... faul ... Wirt ... putzt den Boden

Es wurden 20 Sätze vorgegeben; 10mal war der Genitiv zu ergänzen, wobei die unvollständige NP in der Hälfte der Fälle der Subjekts-NP und in der anderen Hälfte der Objekts-NP folgte. Im Bereich der Präpositionen war in fünf Fällen der Akkusativ und in fünf Fällen die Dativflexion zu ergänzen. ${ }^{26}$

\subsubsection{Ergebnisse}

Auch hier müssen die Ergebnisse für die beiden Kontrollgruppen nicht vorgestellt werden, weil bei ihnen keine Fehler aufgetreten sind. Bereits in Experiment II hatte sich gezeigt, daß auch die ,schlechten" Normalen den Dativ gebrauchen können, - was von den Resultaten von Experiment I her nicht auf der Hand lag. $\mathrm{Daß}$ in Experiment III keine Fehler gemacht wurden, zeigt u.a. daß der in der Alltagssprache unübliche Genitiv von allen Sprechern formal beherrscht wird. ${ }^{27}$

Die Ergebnisse für die agrammatischen Versuchspersonen sind in Tabelle 7 dargestellt. ${ }^{28}$

Da in dieser Aufgabe, ebenso wie in Experiment I, die zu flektierende NP komplexer war $(\mathrm{Art}+\mathrm{Adj}+\mathrm{N})$, wurde in Tabelle 7 wieder die Kategorie ,defektive Kongruenz" eingeführt. Die dort aufgeführten Reaktionen wurden positiv bewertet, da die jeweilige NP als eindeutig und konsistent kasusmarkiert angesehen werden konnte. Die Tatsache, daß beim Genitiv die NPs mit defektiver Kongruenz zunehmen, zeigt, daß die Patienten bewußt sprachlich arbeiten mußten, um an die

in ihrer agrammatischen Sprache selbstverständlich nie auftretende - Form zu gelangen. In (27) werden einige Beispiele von defektiver Kongruenz angegeben: 
Tabelle 7 Ergebnisse für die Agrammatiker (C.B., M.H., H.R.)

\begin{tabular}{|c|c|c|c|c|c|c|c|c|c|c|c|c|c|c|c|c|}
\hline \multirow{5}{*}{ 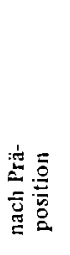 } & \multirow{3}{*}{ ZIEL } & \multicolumn{15}{|c|}{ REAKTION } \\
\hline & & \multicolumn{3}{|c|}{$\mathrm{AKK}$} & \multicolumn{3}{|c|}{ DAT } & \multicolumn{3}{|c|}{ GEN } & \multicolumn{3}{|c|}{$\begin{array}{c}\text { defektive } \\
\text { Kongruenz }\end{array}$} & \multicolumn{3}{|c|}{ uninterpretierbar } \\
\hline & & C.B. & M.H. & H.R. & C.B. & M.H. & H.R. & C.B. & М.H. & H.R. & C.B & M.H. & H.R. & C.B. & H.M. & H.R. \\
\hline & AKK & 100 & 100 & 20 & -- & -- & 80 & $-\infty$ & -- & -- & -- & -- & -- & -- & -- & -- \\
\hline & IDAT & -- & 100 & -- & 100 & -- & 60 & -- & -- & -- & 20 & -- & -- & -- & -- & 40 \\
\hline & GEN & $-\cdot-$ & 20 & 10 & --- & -- & -- & 100 & 80 & 70 & 20 & 30 & 50 & $\cdots$ & -- & 50 \\
\hline
\end{tabular}

Tabelle 8 Ergebnisse für die Paragrammatiker (P.P., H.H.)

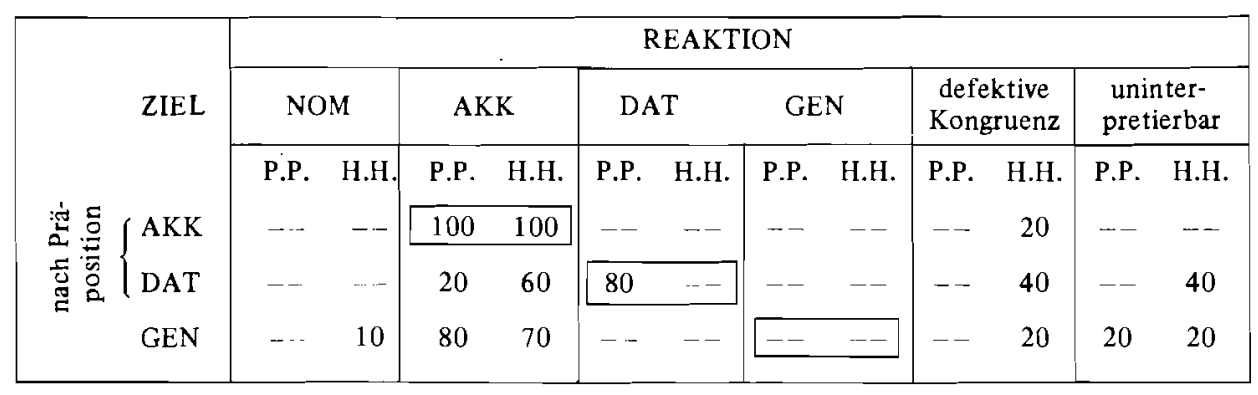

(27) a) Der Besitzer eines kleines Laden entläßt den Lehrling (M.H.)

b) Der Forscher findet den Knochen ein seltenen Vogels (C.B.)

c) Ein rüstiger Rentner segelt zu ein einsamen Strand (C.B.)

d) Der Bischof eines berühmtes Dom begrüßt den Papst (H.R.)

M.H.'s Problem mit dem Dativ zeigt sich auch in dieser Untersuchung wieder. Es spricht einiges dafür, da $ß$ es sich hier um einen prämorbiden Dialektfaktor handelt, der allerdings bei der schlechten Kontrollgruppe nicht durchwegs auftrat. Bemerkenswert ist dagegen, daß M.H. fast immer den Genitiv funktional richtig substituieren kann. Für H.R. gilt dasselbe, mit dem Unterschied, daß sie dazu tendiert, im Bereich von Präpositionen generell den Dativ zu verwenden. Das Bedeutsamste an diesen Ergebnissen ist mit Sicherheit, daß C.B. so gut wie keine Fehler machte. Wie aus der Patientenbeschreibung in 4.1 hervorgeht, handelt es sich bei C.B. um einen Fall von chronischem schweren Agrammatismus. Seine hier demonstrierten linguistischen Fähigkeiten verlangen nach einer Erklärung.

Auch wenn in den Reaktionen von M.H. und H.R. viele Fehler auftraten, so ist doch bemerkenswert, welche Fehler nicht auftraten. Es kam z.B. nie zu der Strategie, den „Null-Kasus“ Nominativ einzusetzen. Es scheinen also ziemlich klare Intuitionen über das Zusammenspiel von Präpositionen und der davon c-kommandierten $\mathrm{NP}$ vorzuliegen.

Kommen wir zu den Ergebnissen der Untersuchung der Paragrammatiker, die in Tabelle 8 dargestellt sind. 
Der Unterschied zu den Agrammatikern ist hier besonders drastisch. Während letztere fast immer die Possessiv-Relation erschliefien. schaffen P.P. und H.H. das in keinem einzigen Fall. Lediglich P.P. verfügt über genügend lexikalische Fähigkeiten, um Kasus durch Präpositionen zuzuweisen. H.H. scheint dagegen kaum Intuitionen über morphosyntaktisch gesteuerte Grammatikalität zu haben. Er produzierte bizarre Fehler, wie etwa in (28):
a) ... sitzt unter eine weiße Balkon
b) ... fällt aus eine schwarzen Koffer
c) ... wohnt bei einen ernste Kaplan
d) ... findet den Knochen eines seltens Vogel

H.H. zeigte sich als relativ hilflos, wenn es darum ging, die eigenen Äußerungen zu kontrollieren und korrigieren.

Zusammenfassend kann man sagen, daß, verglichen mit der subjektiv empfundenen Schwere der Sprachstörung, die metasprachliche Leistungen der Paragrammtiker in scharfem Kontrast zu denen der Agrammatiker stehen. Selbst wenn sie gleich schlecht wie die Agrammatiker abschneiden würden, wäre der dadurch nicht reflektierte Kontrast zur deutlich ..,besseren“ (weil flüssiger und mühelos hervorgebrachten) Spontansprache beachtlich.

\subsection{Zusammenfassung der Ergebnisse}

Unsere Untersuchung zum Kasussystem des Deutschen hat die folgenden vier Hauptresultate erbracht:

I. Die agrammatischen Patienten sind in der Lage, Nominalphrasen der Form $\mathrm{Art}+\mathrm{Adj}+\mathrm{N}$ in sich korrekt zu flektieren. Die paragrammatischen Versuchspersonen machen dabei mehr Fehler. Fälle von defektiver Kongruenz bzw. Uninterpretierbarkeit entstehen bei den Agrammatikern eher durch Auslassungen der zu substituierenden Affixe, bei den Paragrammatikern eher durch das Einsetzen unpassender Affixe, z. B. dadurch, daß ein Nomen mit einem falschen Genus assoziiert wird.

II. Die Agrammatiker zeigen durchgehend, daß sie auch in der Lage sind, die angebotenen NPs dem Kasusparadigma gemäß zu flektieren, d.h. es treten nicht nur Noninative auf, sondern auch in sich wohlgebildete Akkusative, Dative und Genitive. Zumindest in den hier gestellten Aufgaben zeigen die Paragrammatiker weitergehende Defizite. ${ }^{29}$

III. Soweit die Agrammatiker überhaupt einen Kasus X in Abhängigkeit von einem Kasus $\mathrm{Y}$ verarbeiten können, scheinen sie auf wenig erfolgreiche Strategien beschränkt zu sein. Der Tatsache, daß im Deutschen präverbal nicht nur Nominative auftauchen können und postverbal nicht nur Akkusative und Dative etc., wird nicht Rechnung getragen. In den Reaktionen von H.R. gibt es klare Anzeichen dafür, daß bei Satzergänzungsaufgaben eher nach linearer Position vorgegangen wird als nach einer Abhängigkeit vom vorgegebenen Kasus. Dies führt 
dazu, daß die OVS-Fälle „kanonisiert“ werden zu SVS bzw. OVO. Alles was verarbeitet wird, sind möglicherweise die Positionen $\mathrm{VX}$ und $\mathrm{XV}$. Die anderen beiden Patienten benutzen andere Strategien, auf die im Text schon hingewiesen wurde.

Bei der Aufgabe. Doppelobjekt-Konstruktionen in unmarkierter und markierter Konstituentenabfolge zu bearbeiten, läßt sich kein entsprechender Kanonizitätseffekt nachweisen. Bezeichnend dabei ist, daß hier im Fall von C.B. linguistisch klar motivierbare Genitiv-Einsetzungen in der zweiten NP auftreten. bzw. daß die zweite NP aus dem Syntagma herausfällt und (deshalb?) mit dem Nominativ versehen wird.

Während die Paragrammatiker beim Verarbeiten von SVO-versus OVS-Sätzen noch schlechter als die Agrammatiker abschneiden, sind ihre Leistungen bei der Bearbeitung von Doppelobjekt-Konstruktionen deutlich besser. Wir haben keine Erklärung für diesen Unterschied.

IV. Ist die Kasuszuweisung nicht von einem anderen Kasusträger mitbestimmt, sondern einzig von einem lexikalischen Element oder einer invariablen strukturellen Position determiniert, so sind die Agrammatiker sehr erfolgreich. Die Paragrammatiker fallen hier deutlich gegen sie ab. Sie zeigen in etwa dieselben Leistungen wie bei den anderen Aufgaben.

\section{Diskussion}

Kommen wir zurück zu den eingangs vorgestellten Erklärungsansätzen für das Phänomen des Agrammatismus. Liegt dem Agrammatismus eine Syntaxstörung zugrunde, die jegliches syntaktisches Wissen betrifft? Die vorliegende Lintersuchung hat gezeigt, daß diese Hypothese selbst für Patienten mit so schwerem Agrammatismus wie bei C.B. nicht haltbar ist. Beinahe ebenso problematisch ist, Agrammatismus als Störung des Zugriffs zu einem speziellen Vokabular zu sehen. Während es durchaus möglich ist, daß in der Realzeitverarbeitung ("on-line") ein Zugriffsproblem besteht, sind die hier besprochenen Patienten nicht generell unfähig, mit dem Vokabular der geschlossenen Klasse umzugehen. Ihre Reaktionen stellten sich als gezielt und deshalb linguistisch interpretierbar heraus. Die nächste Frage ist: Kann man die These aufrechterhalten, daß es sich um eine reine Performanzstörung handelt? Wir glauben, nein. Ein wesentlicher Grund ist, daß die in unserer Untersuchung verwendeten Aufgaben strikt "off-line" waren, daß den Patienten keine Zeitbeschränkung auferlegt war und daß ilınen sogar geholfen wurde, soweit dies nötig war und soweit es das Untersuchungsziel nicht tangierte. Würde es sich um eine reine Performanzstörung handeln, so sollten die Patienten in diesen Aufgaben eher wie die Kontrollpersonen abschneiden. Tatsächlich zeigten sie zwar mehr strukturelle Fähigkeiten als ihre Spontansprache vermuten ließe, waren aber in allen Aufgaben deutlich schlechter als die normalen Kontrollgruppen. Weder das Extrem einer durchgehenden Syntaxstörung noch das andere Extrem einer reinen 
Performanzstörung (bei erhaltener Kompetenz) scheint haltbar zu sein. Wir werden auf diesen Punkt noch zurückkommen. Alles was die Adaptationshypothese zu den Ergebnissen sagen kann, ist, daß die Patienten im Grunde mehr können als sie in ihrer Spontansprache zeigen und daß die Nichtbenützung dieser Kenntnisse darauf zurückzuführen ist, daß die Patienten bei ihrem Einsatz Schwierigkeiten erwarten. Die Adaptationshypothese sagt aber nichts über die Natur der Störung aus. Fragen wir also nach einer linguistischen Beschreibung, die die beobachteten Unterschiede in den Reaktionen der Agrammatiker erfaßt.

\subsection{Ein linguistischer Erklärungsansatz}

Wir gehen von einer linguistischen Theorie aus, nach der verschiedene Komponenten zusammenspielen, die in sich autonom organisiert sind. Da ist zunächst einmal ein Lexikon, das neben den Lexemen der Sprache und ihrer Bedeutung auch formale Kategorisierungen enthält, z. B. welche Komplementypen selegiert werden etc. Einer dieser Einträge muß im Deutschen auch sein, welchen Kasus ein gegebener Kasuszuweiser (V, P, A) regiert. Die formale Charakterisierung lexikalischer Einträge muß sich auf der Ebene der syntaktischen Phrasen widerspiegeln. Ein Lexem $X^{0}$ muß also an dem Punkt, an dem lexikalische Insertion stattfindet, eine Phrasenstruktur vorfinden, die mindestens so reich ist, daß die obligatorischen Argumente von $X^{0}$ abgelegt werden können. Wir möchten dies an zwei Verben verdeutlichen, deren Lexikoneinträge in (29) skizziert sind:

$$
\begin{array}{lll}
\text { a) rauch: } & \mathrm{V}, & \left(\mathrm{NP}_{\mathrm{akk}}\right) \\
& \theta_{\mathrm{THEME}} \\
\text { b) leg: } & \mathrm{V}, & \mathrm{NP}_{\text {akk }} \mathrm{PP}_{-} \\
& \theta_{\text {THEME }} \theta_{\text {LOCATION }}
\end{array}
$$

(29a) besagt, daß der Stamm rauch ein Verb ist, das optional ein Akkustivobjekt selegiert und daß mit einem solchen Objekt die thematische Rolle ( $\theta$-Rolle) THEME verbunden ist. ${ }^{30}$ (29) b besagt, daß der Stamm leg ein Verb ist, das obligatorisch ein Akkusativobjekt und eine PP mit sich zieht, wobei ersteres die Rolle THEME und letzteres die Rolle LOCATION bekommt. Wie (30) zeigt, muß eine Phrasenstruktur, die (29a) absorbieren soll, nicht so reich sein wie eine, die (29b) absorbieren soll:

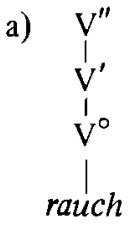

b)

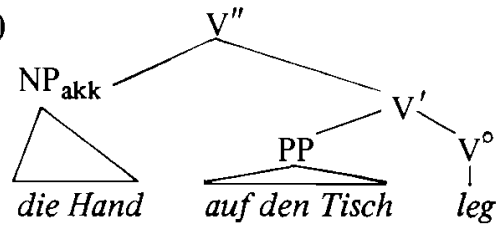

Möglicherweise ist schon im Lexikon festgelegt, daß bei intransitiven Verben $\mathrm{V}^{\min }\left(=\mathrm{V}^{0}\right)$ identisch mit $\mathrm{V}^{\max }\left(=\mathrm{V}^{\prime \prime}\right)$ ist. Um aber transitive Verben, Präpositionen etc. in ein Syntagma einzusetzen, muß das Syntagma eine gewisse minimale Ausdehnung besitzen. 
Die agrammatischen Patienten C.B., M.H. und H.R. haben in den Experimenten gezeigt, daß sie über wichtige lexikalische Informationen verfügen. So ist z. B. die Genusinformation mit Sicherheit bei jedem Nomen des Deutschen lexikalisch kodiert. Würden die Agrammatiker über diese Information nicht verfügen, so würden sie bei Aufgaben wie in den Experimenten I und III wesentlich schlechter abschneiden; (Resultate wie *eine schlanke Boot wurden als uninterpretierbar eingestuft). Man könnte einwenden, daß der Erfolg bei der Genusselektion dadurch garantiert war, daß zumindest in Experiment I meistens NPs verwendet wurden, mit denen ein natürliches Geschlecht assoziiert ist, und daß dies eine Erleichterung der Aufgabe darstellte. Für das dritte Experiment und die dabei verwendeten NPs nach Präpositionen trifft dies jedoch nicht zu. Aber gerade hier passierten kaum Genusfehler. Es wurde in einer anderen Untersuchung mit denselben drei Patienten festgestellt, daß sie mit bemerkenswerter Sicherheịt über Genusinformationen verfügen. Die Patienten wurden nicht nur aufgefordert, den passenden Artikel zu einem gegebenen einfachen Nomen hinzuzufügen, sondern auch zu morphologisch komplexen Nomina (Derivation und Komposition); dazu muß eine Wortsyntax zur Verfügung stehen, die bestimmt, was der Kopf des komplexen Worts ist. ${ }^{31}$ Entsprechende Fähigkeiten zeigten sie auch in bezug auf Numerus. ${ }^{32}$

Nehmen wir an, daß alle morphologischen Prozesse im Lexikon bzw. in einer zum Lexikon gehörigen generativen Morphologie stattfinden. ${ }^{33}$ Wenn Agrammatiker über klare Lexikoninformationen wie Genus und Numerus (im Deutschen) verfügen, warum sollten sie nicht auch die Kasusformen beherrschen? Die lexikalische Morphologie sagt voraus, daß alle (Kasus-)Formen bereits im Lexikon verfugbar sind. Den Wörtern wachsen also nicht erst in der Phrasensyntax Kasussuffixe, sondern sie werden schon voll flektiert in einen phrasalen Rahmen eingesetzt. Nehmen wir weiter an, daß die Syntax wegen einer schweren Störung auf einfache $X^{\prime}$-Strukturen beschränkt ist, also etwa
a) $\mathrm{N}^{\prime \prime} \rightarrow \operatorname{Art} \mathrm{N}^{\prime}$
$\mathrm{N}^{\prime} \rightarrow(\mathrm{AP}) \mathrm{N}^{0}$
b) $\mathrm{P}^{\prime} \rightarrow \mathrm{P}^{0} \mathrm{~N}^{\prime \prime}$
c) $\mathrm{V}^{\prime \prime} \rightarrow \ldots \mathrm{V}^{\prime}$
$\mathrm{V}^{\prime} \rightarrow \ldots \mathrm{V}^{0}$

Die Regeln in (31) generieren Strukturen, in die lexikalische Elemente projiziert werden können:
a) der Hund; der große Hund
b) gegen den Hund; gegen den großen Hund
c) den Hund füttern; den großen Hund füttern

Solche Phrasen sind zwar nicht gerade typisch agrammatisch, aber sie stellen plausible Idealisierungen dar. Im Idealfall wäre es also so, daß Agrammatismus eine Beschränkung der Sprache auf rein lexikalisch lizensierte Substrukturen ist. Es sei angemerkt, daß Phrasen wie in (32) von einem System generiert werden, das syntak- 
tisch extrem arm ist. Alles was geleistet wird, ist die Transmission bestimmter Merkmale vom $X^{0}$-Kopf auf die Nicht-Kopf-Elemente (Komplemente, Spezifizierer). Die möglichen Syntagmen sind finit und strikt lokal, d.h. Bewegung, Bindung, 'scrambling' und dergleichen treten nicht auf.

Die drei hier studierten Agrammatiker haben gezeigt, daß sie zumindest über Phrasen der Art (32a, b) verfügen. Wenn wir nun die These verteidigen wollen, daß bei Agrammatismus die Syntax lediglich ein Reflex des erhaltenen Lexikons ist, so muß zumindest auf die folgenden beiden Einwände geantwortet werden:

(A) Warum sprechen Agrammatiker nicht in kurzen aber wohlgeformten Phrasen, sondern eher im Telegramınstil? Warum werden die Phrasen eher kleiner oder bleiben ganz weg zugunsten von lexikalisch ausgelösten Inkorporationen vom Typ kuchenessen, autowaschen, etc.?

(B) Warum brechen Agrammatiker dennoch oft aus dem rein lexikalischen Schema aus? Warum benutzen sie manchmal Verb-Zweit-Sätze? Warum haben sie im Experiment kaum Probleme mit dem Genitiv als strukturellem Kasus?

Wenden wir uns zunächst (A) zu. Niemand wird leugnen wollen, daß angesichts einer Sprachstörung die kommunikativen Anforderungen nicht aufhören, zu existieren. Kommunikation volizieht sich aber im wesentlichen dadurch, daß Propositionen ausgetauscht werden. Rein lexikalisch lizensierte Phrasen sind aber gerade nicht in der Lage, Propositionen auszudrücken; sie drücken lediglich Relationen und Eigenschaften aus oder stellen Referenz her. Um Propositionen zu bekommen, muß man über Sätze verfügen. Da Agrammatiker (ebenso wie andere Menschen) in erster Linie zum Kommunizieren gezwungen sind, kann man davon ausgehen, daß ihre Äußerungen als Propositionen und damit als Sätze intendiert sind. Diese Intention hat moglicherweise fatale strukturelle Folgen. Agranmatiker haben mit großser Wahrscheinlichkeit ebenso wie Normale Intuitionen über Prädikationsbeziehungen. Sie werden also in vielen Fällen die erste Konstituente als Subjekt und den Rest als Prädikat auffassen. Ihre eigene Rede besteht bevorzugt dann aus subjektlosen Äußerungen, wenn in der ersten Person gesprochen wird und wenn keine finiten Verbformen benutzt werden. ${ }^{34}$ Unter der plausiblen Annahme, daß die linguistischen Verarbeitungskapazitäten von Agrammatikern rein quantitativ eingeschränkt sind, folgt, daß der Gebrauch einer Prädikationsstruktur zu Lasten einer anderen Struktur (z.B. der prädikatsinternen) geht und umgekehrt. Bei einem bestimmten Schweregrad der Aphasie, die z.B. nur die Verarbeitung von zwei Hauptkonstituenten zuläßt. wären Äußerungen möglich wie [Der Mann] ... [rauchen] oder [eine Pfeife] [rauchen] oder [Mann] ... [Pfeiferauchen] aber kaum [Der Mann] ... [eine Pfeife] [rauchen]. Da Phrasen wie die in (32) für kommunikative Zwecke nicht ausreichen und da es wahrscheinlich Verarbeitungseinschränkungen der oben beschriebenen Art gibt, ist es erklärbar, warum Patienten bei einem entsprechenden Schweregrad der Aphasie ihr 'tacit knowledge' in der Spontansprache nicht umsetzen, sondern es allenfalls unter experimentellen oder therapeutischen Bedingungen zum Vorschein kommen lassen. 
Wenden wir uns nun Einwand (B) zu. Wie können unter einer Beschränkung auf das Lexikon und auf die $\mathrm{X}^{\prime}$-Syntax Verb-Zweit-Strukturen auftreten? Wie können Genitiv-NPs auftreten, die nicht von einem lexikalischen Kopf lizensiert werden? Offenbar haben Agrammatiker einen syntaktischen Parser zur Verfügung, der über ein lexikalisch induzierte Strukturen hinausgeht. Wie auch immer ein post-nominaler Genitiv im Deutschen analysiert wird, er kann nicht durch eine lexikalische Regel ausgelöst sein. Wie die Reaktionen von C.B. in Experiment II und die Reaktionen aller drei Patienten in Experiment III gezeigt haben, stehen ihnen genügend semantische Informationen zur Verfügung, um die Possessionsrelation zu erschliefsen. In einem solchen Fall kann der (syntaktisch defizitäre) Parser in Abhängigkeit von einer NP einer unmittelbar darauffolgenden NP den Genitiv (und eine entsprechende $\theta$-Rolle) zuweisen. Es ist dabei zu beobachten, daß es sich um eine strikt lokale Beziehung handelt.

Für die Erklärung von Verb-Zweit-Sätzen kann man folgende Annahmen vorausschicken: Wenn alle morphologischen Prozesse im Lexikon stattfinden, so sind auch die Flexionssuffixe des Verbs im Lexikon verfügbar. Flexionssuffixe wie -st, - $t$, -en kodieren aber genau diejenigen Merkmale, die in der generativen Gramlmatik INFL zugeschrieben werden. Nach Chomsky (1986) könnte dieBasisstruktur eines deutschen finiten Satzes wie folgt aussehen:

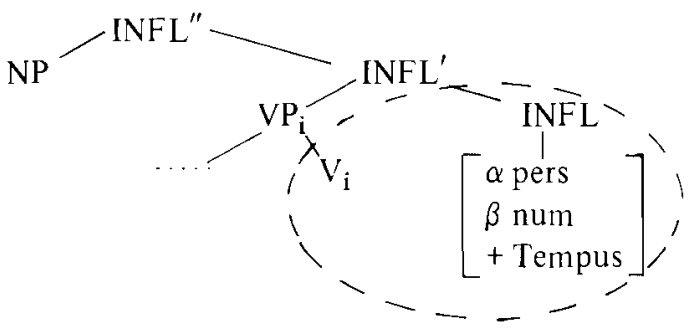

Dies heißt, daß INFL, obwohl es als Merkmalbündel mit einem verbalen Stamm verschmolzen sein muß, auf einer abstrakten Ebene VP als Komplement selegiert. Das Resultat dieser Selektion ist INFL'. Die Subjekt-NP ist dann so etwas wie ein „Spezifizierer“. Die finite Verbmorphologie lizensiert also die Subjektstelle lexikalisch. Agrammatiker, die finite Verben benutzen, äußern aber typischerweise keine Sätze wie der Mann eine Pfeife raucht, sondern Verb-Zweit-Sätze. Das finite Verb geht automatisch an die Spitze einer Struktur wie (33). Die Position, in der das Verb landet, wird in der modernen Syntaxtheorie des Deutschen als eine Mischung aus COMP und INFL angesehen (CONFL). ${ }^{35}$ Nennen wir die Position einfach C. C bildet mit der in (33) gezeigten Struktur eine Projektion C'. Das Vorfeld kann dann wieder als „Spezifizierer" von $C^{\prime}$ aufgefaßt werden. Die Struktur des Hauptsatzes ist dann die in (34) gezeigte: 
(34)

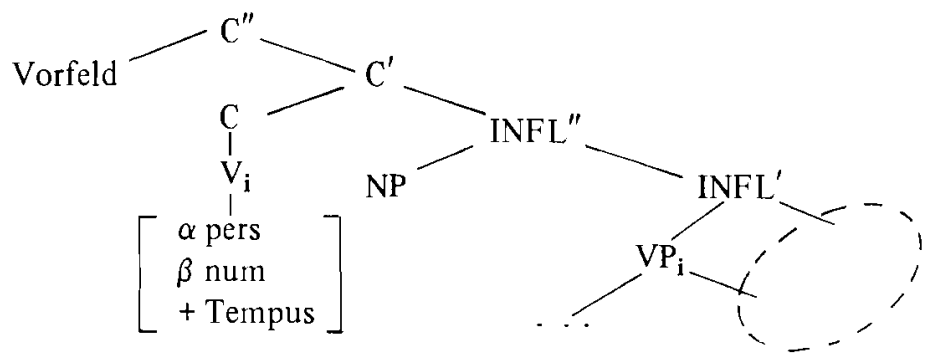

Es ist unklar, ob die Leerstelle, die die Verbbewegung hinterläßt, analog zu anderen Bindungsrelationen in einer Kette $\left(V_{i} \ldots e_{i}\right)$ festgehalten werden soll. Wenn solche Ketten irgendetwas mit der Verarbeitung von Sätzen zu tun haben sollen, spricht nichts dafür. Für den Parser ist es nämlich umso besser, je eher das finite Verb angetroffen wird. ${ }^{36}$

Agrammatiker, die Verb-Zweit-Sätze produzieren, müssen Strukturen wie in (34) wenigstens ausschnittweise zur Verfügung haben. Für die hier vorgestellten Patienten trifft dies zu. Zumindest die im Text gegebenen Beispiele von Bildbeschreibungen belegen das. Daß die Patienten Schwierigkeiten damit haben, geht aus den Ergebnissen von Experiment I hervor. Evidenz liefert auch die Spontansprache von H.R. Wie der in (12) gegebene Ausschnitt zeigt, ist bei Verb-Zweit-Sätzen die Klammer zwischen dem finiten Verb und der abgetrennten Partikel eng, z. B.

a) Ich stehe auf um neun Uhr

b) und gehe hinunter im Wohnzimmer

anstatt ich stehe um neun Uhr auf, und gehe ins Wohnzimmer hinunter. Ganz offenbar hat die Patientin Schwierigkeiten mit dem Satz dann ziehe ich mich um. Ihre Versuche werden in (36) wiedergegeben:
a) ziehe um
b) ziehe ich
c) ziehe ich dann

Es scheint, als ob nur Momentaufnahmen einer normalen Satzverarbeitungssequenz zur Verfügung stünden. (34) impliziert, daß Sätze, in denen das Subjekt im Vorfeld erscheint, eine Bindungsrelation involvieren. Reïn linguistisch spricht einiges dafür. Es ist aber fraglich, ob sie im Verarbeitungssystem eine Rolle spielt. Es gibt psycholinguistische Evidenz, aus der man schließen kann, daß Wh-elemente, die sich auf eine Subjektsspur beziehen, nicht analog zu solchen verarbeitet werden, die sich auf eine Objektsspur beziehen. ${ }^{37}$ Wenn man nicht in die Idee vernarrt ist, daß jeder Schritt in einer transformationell hergestellten Repräsentation mit einem meßbaren Verarbeitungsschritt korrelieren muß, dann stehen entsprechende psycholinguistische Evidenzen (34) nicht notwendigerweise entgegen. Da offensichtlich die Bindung der Objektstelle einen spürbaren Verarbeitungseffekt auslöst, kann man annehmen, daß auch Aphasiker damit Schwierigkeiten haben, während sie einfache SVO-Sätze bewältigen können. 
Ähnliches gilt für die Vorfeldbesetzung im Allgemeinen. Es gibt z. B. keine guten Gründe dafür, daß Satzadverben wie gestern, wahrscheinlich etc. im Mittelfeld (d.h. innerhalb von INFL") entstehen. Es spricht mehr dafür, daßs sie im Vorfeld basisgeneriert werden. Adverben der Art und Weise wie z. B. langsam werden dagegen semantisch vom Verb gesteuert. Die Voraussage ist dann diese: Wenn Agrammatiker eine Syntaxstörung haben, die die Konstruktion von (psychologisch relevanten) Bindungsbeziehungen verhindert, dann sollten sie mit Sätzen vom Typ (37) signifikant weniger Schwierigkeiten haben als mit Sätzen vom Typ (38):

$$
\begin{aligned}
& \text { Gestern hat der Opa im Keller das Holz aufgeschlichtet } \\
& \text { Langsam }_{\mathbf{i}} \text { hat der Opa im Keller } \mathrm{e}_{\mathbf{i}} \text { das Holz aufgeschlichtet }
\end{aligned}
$$

Wie die in (35) und (36) wiederholten Beispiele nahelegen, ist selbst die vergleichsweise leicht gestörte Patientin H.R. kaum dazu in der Lage, mehr als zwei syntaktische Konstituenten zu bearbeiten, die nicht direkte $X^{\prime}$-Reflexe des Lexikons sind. Versuche, diskontinuierliche Konstruktionen zu bauen, scheitern meistens. Das zeigt sich klar in (36), wo man sieht, daß die Benützung von Verb-Zweit bei einem zurückbleibenden Präfix zwar gelingt, aber auf Kosten der Insertion der obligatorischen Argumente und umgekehrt.

Man könnte einwenden, daß die Schwierigkeiten in der Spontansprache auch bezüglich der von uns ,lokal“ genannten Relationen auftreten. Die experimentelle Evidenz deutet aber daraufhin, daß zwischen lokalen und nicht-lokalen Beziehungen dennoch ein Unterschied besteht. Während in Aufgaben wie in Experiment III Jie Schwierigkeiten aus dem Weg geräumt werden können, bleiben sie in Aufgaben wie in Experiment I bestehen.

Unser Schluß ist, daß die hier besprochenen Agrammatiker ein im wesentlichen intaktes Lexikon haben, sowie dazu in der Lage sind, lexikalische Hauptkategorien in $X^{\prime}$-Phrasen zu projizieren. Syntaktische Beziehungen, die darüber hinausgehen, unterliegen starken Beschränkungen, die wiederum mit dem Begriff der Lokalität charakterisiert werden können. Dieser Befund ist mit der These, daß dem Agrammatismus eine Syntaxstörung zugrundeliegt, dann vereinbar, wenn man bereit ist, Syntax als ein System zu sehen, in dem verschiedene Subsysteme (,Module") zusammenwirken. Bei einer solchen Konzeption ist es erwartbar, daß Störungen einzelne Subsysteme betreffen, während sie andere relativ intakt lassen können. Es sei zum Schluß angemerkt, daß es in der aphasiologischen Literatur mittlerweile zahlreiche Befunde gibt, die mit einer solchen Hypothese im Einklang sind. Es ist aus Platzgründen nicht möglich, einen repräsentativen Überblick zu geben oder auch nur einige wichtige Studien in extenso zu diskutieren. Wichtig sind in diesem Zusammenhang beispielsweise die Arbeiten von Grodzinsky. ${ }^{38}$ Dort wird die These vertreten, daß agrammatisches Verstehen linguistisch dadurch charakterisiert werden kann, daß in einer dem Patienten zugänglichen S(urface)-Struktur alle Elemente gelöscht sind, die nicht lexikalisch spezifiziert sind, also z. B. wh- und NP-Spuren. ${ }^{39}$ Ein nicht-linguistisches, default'-Prinzip garantiert dann, daß NPs, denen aufgrund der Spurenlöschung keine $\theta$-Rolle zugewiesen werden kann, einen ,default' ${ }^{`}$ Wert 
erhalten. Grodzinsky kann damit erklären, warum Agrammatiker im Englischen Passivsätze wie The boy was hit by the girl nur auf dem Zufallsniveau verstehen können. Er kann auch erklären, warum sie Objektsrelativsätze schlechter verstehen als Subjektsrelativsätze (The cat that the dog is biting versus the cat that is biting the dog). ${ }^{40}$ Die Charakterisierung des agrammatischen Defizits als Störung der Spurenbindung, korrekt oder nicht, bedeutet natürlich nichts anderes als daß globale syntaktische Beziehungen nicht mehr verarbeitet werden können. Wir sind mit den Details von Grodzinskys Erklärung des Agrammatismus nicht einverstanden, möchten aber betonen, dałs wir den Ansatz prinzipiell für vielversprechend halten.

Von einem völlig anderen Standpunkt aus hat Tyler (1985) einen Fall von Agrammatismus, D.E.. studiert. Tyler hat ein 'monitoring' Paradigma verwendet, bei dem der Patient unter drei verschiedenen Bedingungen in einem Satz ein Zielwort lokalisieren mußte. Wie Marslen-Wilson \& Tyler (1980) gezeigt haben, reagieren sprachgesunde Versuchspersonen bei normalen Sätzen am effektivsten, d.h. schnellsten, bei semantisch abweichenden Sätzen weniger effektiv und bei „Wortsalat"-Sätzen am ineffektivsten. Wenn das Zielwort an verschiedenen Stellen im Satz auftaucht, zeigt sich, daß sich die Reaktionszeiten (RZ) progressiv vermindern, und zwar bei normalen wie bei semantisch abnormalen Sätzen. Tyler führt das darauf zurück, dafs die Versuchsperson eine syntaktische Struktur des Wahrgenommenen erstellt. Bei „Wortsalat"-Sätzen tritt so ein Positionseffekt nicht ein. Interessanterweise zeigte sich bei D.E. bei semanisch abnormalen Sätzen kein Positionseffekt, während ein solcher bei normalen Sätzen vorhanden war. Tyler interpretiert diesen Befund dahingehend, daß D.E. keine vollständige syntaktische Repräsentation des Inputs herstellen kann und (deshalb) auf den Faktor der semantischen Erwartbarkeit angewiesen ist. In einem weiteren Experiment konnte Tyler nachweisen, dafi D.E. für Subkategorisierungsverstöße sensibel ist. Beide Ergebnisse deuten darauf hin, und werden von der Autorin auch so interpretiert, daß D.E. wohl in der Lage ist, lokale syntaktische Relationen in Realzeit aufzuspüren, aber nicht, eine globale syntaktische Repräsentation zu erstellen. Tyler schreibt

"What we need is experimental data contrasting the on-line construction of local syntactic phrases with the development of larger scale syntactic organizations."

Wir möchten dem hinzufügen, daß die Aphasiologie darüberhinaus Theorien braucht, die in der Lage sind, naive Konzepte von Syntax (Semantik, Morphologie usw.) durch wissenschaftliche zu ersetzen. Wenn dieser Aufsatz wenigstens in Ansätzen gezeigt hat, daß dies bei der Beschäftigung mit Sprachpathologie von Nutzen sein kann, sind wir zufrieden.

\section{Anmerkungen}

1 siehe Miceli et al. (1983).

2 Dysarthrien treten in verschiedenen Formen auf. Es sei hier nur gesagt, daß Dysarthrien die Sprechmotorik beeinträchtigen, aber in ihrer Reinform das höher kognitive Sprachsystem (z. B. Intuitionen über phonemische Strukturen) nicht berühren. 
3 z. B. Huber et al. (1982).

4 Man kann sich darüber in jedem Standardwerk informieren, z. B. Lesser (1978), Poeck (1982), Friederici (1984).

5 Levelt (1970), Zurif \& Caramazza (1976).

6 Saffran, Schwartz \& Marin (1980).

7 Es sei aber darauf hingewiesen, daß die Bradley'schen Ergebnisse in verschiedenen Studien, auch für andere Sprachen als dem Englischen, nicht repliziert werden konnten.

8 siehe Garrett (1980) und auch Leuninger (in diesem Band)

9 Weitere Diskussion zu diesem Thema, speziell mit Bezug auf verschiedene Typen von Präpositionen, tindet sich in Friederici (1981), (1982) und (1985).

10 Kean (1977) bespricht u.a. Voraussagen ihrer Theorie für das Deutsche und bemerkt, daß Verben im Infinitiv realisiert werden sollten, also trinken statt trinkt etc. Das ist natürlich inkonsistent mit der Theorie, da schon der Stamm trink das minimale phonologische Wort darstellt.

11 siehe Lenneberg (1972: 267 ff.).

12 siehe Heeschen (1985), Kolk \& van Grunsven (1985).

13 Heeschen (1985: 241).

14 Damit kann z. B. erklärt werden, warum im Englischen die Objektskomplemente von help und see beide durch NP-Bewegung verschoben werden können, während im Deutschen eine Kasusasymmetrie besteht:

(i) a) The dog was helped

b) The dog was seen

(ii) a) Dem/*der Hund wurde geholfen

b) ${ }^{*}$ Den/der Hund wurde gesehen

Siehe dazu Haider (1985), wo die Kasusalternation im Rahmen von Kasusabsorption behandelt wird. Bei Adjazenz zu einem ditransitiven Verb scheint es im Englischen Residuen des Dativs zu geben (We gave John a book versus We gave the book to John). Anders als im Deutschen ist dieser Kasus aber an eine bestimmte $\theta$-Rolle geknüpft.

15 Damit wird aber der Genitiv in Fällen wie die Schwester des Mannes ausgeschlossen, was uns unglücklich erscheint.

16 Es gibt gute Gründe, keine transformationelle Besetzung anzunehmen für Fälle, bei denen ein flektierender Quantor an der Argumentstelle verbleibt, z. B.

(i) Bauern haben wir keinen eingeladen

(ii) Bauern sind alle eingeladen worden

Gegen Extraktion sprechen die folgenden Beispiele (siehe Bayer (in Vorb.)):

(iii) Hemd hatte er keines an

(iv) *Er hatte keines Hemd an

17 siehe z. B. Koster (1975).

$18 \mathrm{Ob}$ das durch Bindung an eine leere Kategorie bzw. Bewegung erreicht wird oder durch den Einsatz von komplexen (funktorkomponierten) Kategorien einer Kategorialgrammatik (Steedman 1985) oder durch sog. 'slash categories' der GPSG (Gazdar et al. 1985), ist hier unerheblich.

19 Bei Sätzen wie (i) ergibt sich kein Problem:

(i) Das Kind haben die Frauen gefüttert

Ein deterministischer Parser im Sinne von Marcus (1980) kann nämlich eine begrenzte Zahl von Konstituenten aufsammeln ('look ahead device'), bevor die globale syntaktische Analyse beginnt. Schwierigkeiten für einen solchen Parser könnten sich aber einstellen sobald mehr lexikalisches Material zwischen die in (i) nicht kongruierenden Konstituenten tritt; so müßste $z$. B. in 
(ii) Das Kind von vorgestern, das in einen rostigen Nagel getreten war, haben die Frauen gefüttert

zumindest die Nominativ/ $\theta$-Zuweisung solange zurückgehalten werden, bis die komplexe NP verarbeitet ist. Dies widerspricht aber unserer Ansicht nach der zentralen Idee eines deterministischen Parsers.

20 siehe de Bleser (1987), wo aphasiologische Traditionen in Deutschland diskutiert und mit neueren theoretischen Entwicklungen in Beziehung gesetzt werden; siehe auch Heeschen (1985).

21 Es sei aber angemerkt, daß der AAT das Sprachverständnis nur sehr kursorisch, d.h. ohne Bezug auf schwierige syntaktische Konstruktionen, prüft.

22 Wir konnten diesen Dialektfragen nicht weiter nachgehen, weil es zum Aachenerischen keine Grammatik gibt.

23 Es sei darauf hingewiesen, daß C.B. oft nicht in der Lage war, sich strikt an die Aufgabenstellung zu halten, d.h. nur definite NPs zu bilden. C.B. bildete die NPs nach Belieben auch mit ein... und zuweilen sogar mit dem Possessivpronomen sein... Es ist klar, daß unser Anliegen dadurch nicht gestört wurde.

24 Wegen der geringen Zahl von Stimuli ist es nicht ratsam, diesen Lerneffekt statistisch zu demonstrieren $z$ u versuchen.

25 Man könnte argumentieren, daß die Aufgaben in Experiment II keine diskontinuierlichen Abhängigkeiten involvierten. Demnach müßte eine Doppelobjekt-Konstruktion wie widmet dem Sträfling den Krimi, ignoriert man Verb-Zweit, so analysiert werden, als inkorporiere das Verb die Dativ-NP. Dieser Komplex müßte dann der ,äußeren“ NP den Akkusativ zuweisen. Marginal tolerierbare Vorfeldbesetzungen wie Den Krimi gewidmet hat er doch dem Sträfling sprechen eher gegen eine solche Lösung.

26 Es konnte aus bestimmten Gründen nicht gewährleistet werden, daß ausschließlich die Präposition den Kasus regiert; einige Präpositionen regieren, je nachdem, ob sie mit einem Verb mit Bewegungsaspekt oder mit Zustandsaspekt auftreten, entweder den Akkusativ oder den Dativ: Er läuft in den Wald versus Er läuft im Wald (herum). Präpositionen, die von sich aus mehere Kasus regieren können, wie z. B. wegen, wurden nicht verwendet.

27 Der Genauigkeit halber sei darauf verwiesen, daß im Aachenerischen und eng damit verwandten Dialekten der Genitiv bei Zeitangaben wie des Morgens, des Mittags etc. verwendet wird, aber nicht in Possessivkonstruktionen.

$28 \mathrm{Da}$ es keine interessanten Unterschiede bezüglich der Position der Genitiv-NP gab, wurde in der Tabelle auf eine entsprechende Distinktion verzichtet.

29 H.H. produzierte beispielsweise keinen Genitiv. Wir meinen nicht, daß das beweist, daß H.H. keine Genitivformen in seinem linguistischen System hat, sondern, daß er sein eventuell vorhandenes Wissen nicht ausnützen kann.

30 THEME ist dabei das Objekt, das einer Transition unterworfen ist. Siehe Jackendoff (1972, 2. Kap.).

31 siehe Selkirk (1982), Toman (1983), Höhle (1985) unter anderen.

32 In de Bleser and Bayer (1986) wird gezeigt, daß morpholexikalische Prozesse autonom ablaufen können, d.h. weder einen Zugriff zur Phrasensyntax noch zur Bedeutung voraussetzen. Die morphologischen Fähigkeiten der hier diskutierten agrammatischen Patienten werden detailliert dargestellt in de Bleser \& Bayer (erscheint).

33 siehe Lieber (1980) und Kiparsky (1982).

34 vgl. dazu C.B.'s Äußerungen (8) mit M.H.'s und H.R.'s Äußerungen (10) und (12).

35 siehe Thiersch (1978), den Besten (1982), Platzack (1983), Bayer (1984), Torris (1984), Koopman (1984).

36 siehe dazu Bach et al. (in diesem Band). 
37 siehe u. a. Stowe (1984). Stowe zieht daraus den Schluß, daß in Sätzen wie Who comes? das Fragemorphem who in situ bleibt. Analog argumentiert auch Gazdar (1981), wo auf S. 172 noch weitere einschlägige psycholinguistische Literatur zitiert wird. Siehe auch Chomsky (1986) über 'vacuous movement'. Daß die Subjekts-NP nicht prinzipiell in situ bleibt, wird direkt belegt durch die Syntax des Bairischen, wo w-Wörter links vom Complementizer landen dürfen, z. B. Ich weiß nicht [wer daß uns noch helfen könnte], der Mann [der wo draußen wartet]. Siehe Bayer (1984).

38 siehe Grodzinsky (1986).

39 Unglücklicherweise fallen darunter, nimmt man wie Grodzinsky die Government and Binding Theorie an, auch PRO und pro. Agrammatiker scheinen aber keine herausragenden Probleme beim Verstehen von Kontrollsätzen zu haben. Siehe dazu Lonzi \& Zanobio (1983).

40 Ein unangenehmer Nebeneffekt dieser Analyse ist, daß für sog. 'truncated passives' das Default-Prinzip vorhersagt, daß das Oberflächensubjekt immer als AGENS verstanden wird. Dies erscheint uns höchst unplausibel.

\section{Literatur}

Bach, E., C. Brown \& W. D. Marslen-Wilson (in diesem Band): „Gekreuzte und geschach telte Abhängigkeiten im Deutschen und im Niederländischen: Eine psycholinguistische Untersuchung".

Bayer, J. (1984): "COMP in Bavarian syntax". The Linguistic Review 3, 209-274.

Bayer, J. (in Vorb.): The syntax of scalar particles and so-called "floating quantifiers". Ms. Max-Planck-Institut für Psycholinguistik, Nijmegen.

Bayer, J. \& R. de Bleser (in Vorb.): "On so-called grammaticality judgments of agrammatic speakers."

den Besten, H. (1982): "On the interaction of root transformations and lexical deletive rules." In W. Abraham (Hrsg.), On the Formal Syntax of the Westgermania, John Benjamins V. B., Amsterdam.

de Bleser, R. (1987): "From agrammatism to paragrammatism: German aphasiological traditions and grammatical distrubances." Cognitive Neuropsychology. 4, 187-256.

de Bleser, R. \& J. Bayer (1986): "German word fomation and aphasia." The Linguistic Review $5,1-40$.

de Bleser, R. \& J. Bayer (erscheint): "On the role of inflectional morphology in agrammatism". In M. Hammond \& M. Noonan (Hrsg.), Theoretical Morphology. Academic Press, New York. Bradley, D. C. (1978): Computational Distinction of Vocabulary Type. PhD diss. MIT.

Bradley, D. C., M. F. Garrett \& E. B. Zurif (1980): "Syntactic deficits in Broca's Aphasia." In D. Caplan (Hrsg.), Biological Studies of Mental Processes. MIT Press, Cambridge, Mass.

Caplan, D. (im Druck): Neurolinguistics and Linguistic Aphasiology: An Introduction. Cambridge University Press. Cambridge.

Chomsky, N. A. (1981): Lectures on Government and Binding. Foris, Dordrecht.

Chomsky, N. A. (1986): Barriers. MIT Press, Cambridge, Mass.

Chomsky, N. A. \& M. Halle (1968): The Sound Pattern of English. Harper and Row, New York.

Friederici, A. D. (1981): "Production and comprehension of prepositions in aphasia." Neuropsychologia 19, 191-199.

Friederici, A. D. (1982): "Syntactic and semantic processes in aphasic deficits: The availability of prepositions." Brain and Language 15, 249-258.

Friederici, A. D. (1984): Neuropsychologie der Sprache. Kohlhammer, Stuttgart.

Friederici, A. D. (1985): "Levels of processing and vocabulary types: Evidence from on-line comprehension in normals and agrammatics." Cognition 19, 133-166.

Garrett, M. F. (1980): "Levels of processing in sentence production." In B. Butterworth (Hrsg.), Language Production, Bd. I. Academic Press, London. 
Gazdar, G. (1981): "Unbounded dependencies and coordinate structure." Linguistic Inquiry $12,155-184$.

Gazdar, G., E. Klein, G. Pullum \& I. Sag (1985): Generalized Phrase Structure Grammar. Blackwell, Oxford.

Grodzinsky, Y. (1986): "Language deficits and the theory of syntax." Brain and Language 27.

Haider, H. (1985): "The case of German." In J. Toman (Hrsg.), Studies in German Grammar. Foris, Dordrecht.

Heeschen, C. (1985): "Agrammatism versus paragrammatism: A fictitious opposition." In M. L. Kean (Hrsg.), Agrammatism. Academic Press, New York.

Höhle, T. N. (1985): "On composition and derivation: The constituent structure of secondary words in German." In J. Toman (Hrsg.), Studies in German Grammar. Foris, Dordrecht.

Huber, W., K. Poeck, D. Weniger \& K. Willmes (1982): Der Aachener Aphasie Test. Hogrefe, Göttingen.

Jackendoff, R. (1972): Semantic Interpretation in Generative Grammar. MIT Press, Cambridge, Mass.

Kiparksy, P. (1982): "From cyclic phonology to lexical phonology." In H. van der Hulst \& N. Smith (Hrsg.), The Structure of Phonological Representations Bd. I. Foris, Dordrecht.

Kolk, H. J. \& M. van Grunsven (1985): "Agrammatism as a variable phenomenon." Cognitive Neuropsychology 2, 347-384.

Koopman, H. (1984): The Syntax of Verbs. Foris, Dordrecht.

Koster, J. (1975): "Dutch as an SOV-language." Linguistic Analysis 1, 111-136.

Lenneberg, E. H. (1972): Biologische Grundlagen der Sprache. Suhrkamp, Frankfurt am Main.

Lesser, R. (1978): Linguistic Investigations of Aphasia. Edward Arnold, London.

Leuninger, H. (in diesem Band): „Das ist wirklich ein dickes Stück: Überlegungen zu einem Sprachproduktionsmodell."

Levelt, W. J. M. (1970): "A scaling approach to the study of syntactic relations." In G. B. Flores d'Arcais \& W. J. M. Levelt (Hrsg.), Advances in Psycholinguistics, North Holland, Amsterdam.

Lieber, R. (1980): On the Organization of the Lexicon. PhD diss., MIT.

Linebarger, M. C., M. F. Schwartz \& E. M. Saffran (1983): "Sensitivity to grammatical structure in so-called agrammatism." Cognition 13, 361-392.

Lonzi, L. \& E. Zanobio (1983): "Syntactic comprehension in language responsible cognitive structure: Neurological evidence." Brain and Language 18, 177-191.

Marcus, M. (1980): A Theory of Syntactic Recognition for Natural Language. MIT Press, Cambridge, Mass,

Marslen-Wilson, W. D. \& L. Tyler (1980): "The temporal structure of spoken language understanding." Cognition $8,1-71$.

Miceli, G., A. Mazzucchi, L. Menn \& H. Goodglass (1983): "Contrasting cases of Italian agrammatic aphasia without comprehension disorder." Brain and Language 19, 65-97.

Platzack, C. (1983): "Germanic word order and the COMP/INFL parameter." Working Papers in Scandinavian Syntax 2.

Poeck, K. (1982) (Hrsg.): Klinische Neuropsychologie. Thieme, Stuttgart.

Saffran, E. M., M. F. Schwartz \& O. S. M. Marin (1980): "The word order problem in agrammatism II. Production." Brain and Language 10, 263-280.

Selkirk, L. O. (1982): The Syntax of Words. MIT Press, Cambridge, Mass.

Steedman, M. (1985): "Dependence and coordination in the grammar of Dutch and English." Language 61, 523-568.

Stowe, L. A. (1984): "A subject/object asymmetry in parsing." Proceedings of NELS 14. University of Massachusetts/Amherst.

Thiersch, C. L. (1978): Topics in German Syntax. PhD diss. MIT.

Toman, J. (1983): Wortsyntax: Eine Diskussion ausgewählter Probleme deutscher Wortbildung. Niemeyer, Tübingen. 
Torris, T. (1984): Syntactic Configurations and Discontinuous Dependencies in Contemporary. German. Erscheint (in Frz.) in der Reihe .,Linguistische Arbeiten", Niemeyer, Tübingen.

Tyler, L. (1985): "Real-tıme comprehension processes in agrammatism: A case study," Brain and Language 26, $259 \cdot 275$

Weigl, E. \& M. Bierwisch (1970): "Neuropsychology and linguistics: Topics of common le $^{2-}$ search." Foundations of Langugge 6, 1--18.

Zurif, E. B. \& A. Caramazza (1976): "Psycholinguistic structures in aphasia: Studies in syntax and semantics." In H. Whitaker \& H. A. Whitaker (Hrsg.), Studies in Neurolinguistics, Bd. I, Academic Press. Neu lork. 